\title{
ARTICLE OPEN \\ FX5, a non-steroidal glucocorticoid receptor antagonist, ameliorates diabetic cognitive impairment in mice
}

Dan-yang Zhu ${ }^{1}$, Jian $\mathrm{Lu}^{1}$, Rui Xu ${ }^{1}$, Juan-zhen Yang ${ }^{1}$, Xiang-rui Meng ${ }^{2}$, Xing-nan Ou-Yang ${ }^{1}$, Qiu-ying Yan ${ }^{1}$, Rui-fang Nie ${ }^{1}$, Tong Zhao ${ }^{1}$, Yi-di Chen ${ }^{1}$, Yin Lu ${ }^{1}$, Yi-nan Zhang ${ }^{1}$, Wen-jun $\mathrm{Li}^{1}$ and Xu Shen ${ }^{1}$

Diabetic cognitive impairment (DCI) is a common diabetic complication characterized by learning and memory deficits. In diabetic patients, hyperactivated hypothalamic-pituitary-adrenal (HPA) axis leads to abnormal increase of glucocorticoids (GCs), which causes the damage of hippocampal neurons and cognitive impairment. In this study we investigated the cognition-improving effects of a non-steroidal glucocorticoid receptor (GR) antagonist 5-chloro-N-[4-chloro-3-(trifluoromethyl) phenyl]thiophene-2sulfonamide (FX5) in diabetic mice. Four weeks after T1DM or T2DM was induced, the mice were administered FX5 (20, $40 \mathrm{mg} \cdot \mathrm{kg}^{-1} \cdot \mathrm{d}^{-1}$, i.g.) for 8 weeks. Cognitive impairment was assessed in open field test, novel object recognition test, Y-maze test, and Morris water maze test. We showed that FX5 administration significantly ameliorated the cognitive impairments in both type 1 and 2 diabetic mice. Similar cognitive improvement was observed in diabetic mice following brain GR-specific knockdown by injecting AAV-si-GR. Moreover, AAV-si-GR injection occluded the cognition-improving effects of FX5, suggesting that FX5 functioning as a non-steroidal GR antagonist. In PA-treated primary neurons (as DCI model in vitro), we demonstrated that FX5 (2, 5 , $10 \mu \mathrm{M})$ dose-dependently ameliorated synaptic impairment via upregulating GR/BDNF/TrkB/CREB pathway, protected against neuronal apoptosis through repressing GR/PI3K/AKT/GSK3 $\beta$-mediated tauopathy and subsequent endoplasmic reticulum stress. In LPS-treated primary microglia, FX5 dose-dependently inhibited inflammation through GR/NF-KB/NLRP3/ASC/Caspase-1 pathway. These beneficial effects were also observed in the hippocampus of diabetic mice following FX5 administration. Collectively, we have elucidated the mechanisms underlying the beneficial effects of non-steroidal GR antagonist FX5 on DCl and highlighted the potential of FX5 in the treatment of the disease.

Keywords: diabetes; diabetic cognitive impairment; glucocorticoid receptor antagonist; FX5; learning and memory; hippocampus; synaptic impairment; neuronal apoptosis; inflammation

Acta Pharmacologica Sinica (2022) 43:2495-2510; https://doi.org/10.1038/s41401-022-00884-9

\section{INTRODUCTION}

Diabetic cognitive impairment $(\mathrm{DCl})$ is a chronic diabetic complication with complicated pathogenesis [1]. DCl is characterized by deficit of learning and memory abilities. According to the estimates of the International Diabetes Federation, 463 million people are living with diabetes and the number is expected to rise to 700 million by 2045 [2]. DCl has affected about $30 \%$ of diabetic patients, and DCl patients are more likely to suffer from Alzheimer's disease (AD) compared with common population [3]. Currently, clarifying $\mathrm{DCl}$ pathogenesis and performing mechanism-based anti-DCl target and drug research have become hotspots in the field of contemporary diabetes research.

$\mathrm{DCl}$ is a multi-factor pathogenic process in patients with both type 1 (T1DM) and type 2 (T2DM) diabetic mellitus, although their mechanisms of cognitive deficits tend to be slightly different. In T1DM pathology, impaired insulin signaling due to insulin deficiency causes inflammation, oxidative stress, apoptosis [4], and neurotrophic factor suppression [5] resulting in synaptic plasticity and integrity impairment and further neuronal loss [6]. By contrast, T2DM has a more complicated pathogenesis, in that impaired insulin signaling induced by insulin resistance impairs tyrosine phosphorylation of IRS leading to inhibition of PI3K/AKT and GSK3 $\beta$ signaling [7] and further activation of tau hyperphosphorylation [8]. Moreover, in addition to impaired insulin signaling, abnormal cholesterol metabolism [9] and excessive activation of hypothalamic-pituitary-adrenal axis (HPA) also aggravates cerebral microvascular lesions [10] and induces neuronal apoptosis, eventually resulting in DCl of T1DM and T2DM.

Glucocorticoids (GCs, e.g., cortisone and hydrocortisone) [11] are a group of hormones secreted by adrenal gland in cellular, molecular, and physiologic network of the body [12]. Under normal physiological conditions, negative feedback is driven by GCs on HPA axis to normalize GCs secretion [13]. However, HPA axis is hyperactivated and circulating cortisol is increased in patients with diabetes [14, 15]. Similarly, adrenal GCs are also elevated in diabetic rodents [16]. GCs render deleterious effects on hippocampus [17], including mitochondrial dysfunction [18], cell cycle arrest and cell death [19]. In addition, abnormal excess of GCs may also induce neuronal atrophy and synaptic dysfunction (e.g., disturbance of integrity of synaptic

\footnotetext{
'Jiangsu Key Laboratory for Pharmacology and Safety Evaluation of Chinese Materia Medica and State Key Laboratory Cultivation Base for TCM Quality and Efficacy, Nanjing University of Chinese Medicine, Nanjing 210023, China and ${ }^{2}$ Faculty of Art and Science, Queens University, Kingston, ON K7L 3N6, Canada Correspondence: Yi-nan Zhang (yinanzhang@njucm.edu.cn) or Wen-jun Li (liwenjun@njucm.edu.cn) or Xu Shen (xshen@njucm.edu.cn) These authors contributed equally: Dan-yang Zhu, Jian Lu
} 
skeleton) by upregulation of tau hyperphosphorylation [20] and suppression of brain-derived neurotrophic factor (BDNF) [21]. Moreover, chronic GCs exposure increases inflammation in the brain [22]. All results have thus addressed the potency of GCs in the regulation of cognitive impairment.

GCs function by binding glucocorticoid receptor (GR) or mineralocorticoid receptor (MR), two members of nuclear hormone receptors superfamily [23]. MR expression is restricted to a few tissues and GR is ubiquitously expressed in the brain [24]. Currently, several GR antagonists have been reported with beneficial effects on $\mathrm{DCl}$. For example, mifepristone as a steroidal GR antagonist ameliorated cognitive impairment in STZ-induced T1DM rats [25], but its long-term administration may cause side effects like hypokalemia [26], adrenal insufficiency [27], and uterine cramping [28]. Therefore, non-steroidal GR antagonist discovery attracts much attention and reports have been published on the amelioration of non-steroidal GR antagonists on DCl, although the detailed mechanisms are still obscure [29].

In the current work, we determined that small molecule 5-chloro$\mathrm{N}$-[4-chloro-3- (trifluoromethyl)phenyl] thiophene-2-sulfonamide (FX5, Fig. 1a [30]) as a non-steroidal GR antagonist improved learning and memory in both T1DM and T2DM mice. The underlying mechanisms have been intensively investigated by assay against the GR-specific knockdown diabetic mice treated with adeno-associated virus (AAV)-ePHP-si-GR. Our work has strongly addressed the potency of non-steroidal GR antagonist in the amelioration of $\mathrm{DCl}$ and highlighted the potential of FX5 in the treatment of this disease.

\section{MATERIALS AND METHODS}

Materials

All cell culture reagents were purchased from Gibco (Grand Island, New York, USA). Plasmids of pCl-nGFP-C656G, pRL-SV40 and pUASTK-Luc were kindly donated respectively by Dr. Gordon Hager (National Cancer Institute, National Institutes of Health), Dr. J Larry Jameson (Department of Medicine, Northwestern Memorial Hospital) and Dr. Daniel P. Kelly (School of Medicine, Washington University). Other plasmids were available in our own lab. Dimethy 1 sulfoxide (DMSO), Tween-80, 3-(4,5-dimethylthiazol-2-yl)-2,5-diphenyltetrazolium bromide (MTT), DNase, poly-D-lysine (PDL), dexamethasone (Dex), corticosterone (Cort), progesterone (Prog), T0901317 (T090), estradiol (Est), streptozotocin (STZ), secondary anti-rabbit/mouse antibodies, lipopolysaccharide (LPS), and palmitic acid (PA) were purchased from Sigma-Aldrich (St, Louis, MO, USA). RIPA buffer and BCA protein assay kits were purchased from Beyotime (Shanghai, China). Protease inhibitor cocktail and phosphatase inhibitor cocktail were purchased from Thermo Fisher Scientific (Waltham, MA, USA). sinegative control (si-NC) and si-GR plasmids were purchased from GenePharma (Shanghai, China). Lipofectamine 2000 was purchased from Invitrogen (Carlsbad, CA, USA).

\section{Cell culture}

Pregnant mice and newborn mice were purchased from Vital River Laboratory Animal Technology Co (Beijing, China). All animal experiments were performed following the institutional ethical guidelines on animal care of Nanjing University of Chinese Medicine.

Primary neurons were prepared from embryonic day 16 to 18mouse brains. The brain tissues were minced into small pieces, digested with D-Hank's buffer containing $0.125 \%$ trypsin and 200 $\mathrm{U} / \mathrm{mL}$ DNase, and incubated for $15 \mathrm{~min}$ at $37^{\circ} \mathrm{C}$. Digestion was stopped by adding $4 \mathrm{~mL}$ DMEM supplemented with $10 \% \mathrm{FBS}$. The cell fluid was then diluted at a density of $6 \times 10^{5}$ cells $/ \mathrm{mL}$ on PDLcoated cell culture plates. After $6 \mathrm{~h}$, the medium was replaced by neurobasal medium supplemented with $2 \%$ B27, $0.5 \mu \mathrm{M}$-glutamine and $50 \mathrm{U} / \mathrm{mL}$ penicillin-streptomycin [31].

Primary microglia were prepared from newborn mice within $24 \mathrm{~h}$. The brain tissues were minced into small pieces, and digested with Hank's buffer containing 0.25\% trypsin and $200 \mathrm{U} /$ $\mathrm{mL}$ DNase. The cell suspension was incubated for $10 \mathrm{~min}$ at room temperature, while digestion was stopped by adding $4 \mathrm{~mL}$ DMEM supplemented with $10 \%$ FBS. Then, the dissociated cells were cultured in DMEM/F12 supplemented with $10 \%$ FBS and $50 \mathrm{U} / \mathrm{mL}$ penicillin-streptomycin using a PDL-coated $75 \mathrm{~cm}^{2}$ flask at a density of $2 \times 10^{5}$ cells $/ \mathrm{cm}^{2}$. After 7 days, the microglia were dissociated by shaking the flasks several times and harvested by centrifugation at $1500 \mathrm{r} / \mathrm{min}$ for $10 \mathrm{~min}$, followed by dilution at a density of $5 \times 10^{4}$ cells $/ \mathrm{mL}$ on PDL-coated cell culture plates [31].

Human embryonic kidney HEK293T cells and U2OS/GR-GFP cells were cultured in DMEM supplemented with $10 \%$ FBS and $100 \mathrm{U} /$ $\mathrm{mL}$ penicillin-streptomycin.

All cells were cultured in a humidified incubator with $5 \% \mathrm{CO}_{2}$ at $37^{\circ} \mathrm{C}$.

Mammalian transactivation assay

Mammalian transactivation assay was carried out to detect the antagonistic effect of FX5 on GR transcriptional activity according to the reported approach [32]. Briefly, HEK293T cells were seeded in 48well plates at a density of $5 \times 10^{4}$ cells/well and cultured overnight. Cells were then co-transfected with plasmids of pCI-nGFP-C656G, pGL3-GRE-Luc, and pRL-SV40 by calcium phosphate cell transfection kit (Beyotime, Shanghai, China). After $6 \mathrm{~h}$, cells were incubated with Dex $(10 \mathrm{nM})$ or/and FX5 $(10 \mu \mathrm{M})$ for $24 \mathrm{~h}$. Finally, firefly and Renilla luciferase activities were measured by dual-luciferase reporter assay system kit (Promega, Madison, WI, USA).

The effect of FX5 on other nuclear receptors MR, progesterone receptor (PR), liver $X$ receptor $\beta(L X R \beta)$, and estrogen receptor $\beta$ $(E R \beta)$ were also detected by using mammalian transactivation assay.

Nuclear translocation assay

U2OS/GR-GFP cells were seeded in 96-well plates at a density of $1 \times 10^{4}$ cells/well and cultured overnight. The cells were then incubated with PA $(0.2 \mathrm{mM})$ or/and different concentrations of FX5 $(2,5$, or $10 \mu \mathrm{M})$ for $24 \mathrm{~h}$. After incubation, cells were immobilized with $4 \%$ paraformaldehyde, and nucleus was dyed with Hoechst 33342 (Solarbio, Beijing, China). Fluorescence pictures were obtained using Leica fluorescence microscope (Leica Microsystems, Nussloch, Germany). The ratio of GR nuclear translocation was calculated by ImageJ software.

MTT assay

Viability of the cultured cells was determined by MTT assay [33]. Briefly, primary neurons were seeded in 96-well plates at a density of $1 \times 10^{5}$ cells/well and cultured overnight. The cells were then incubated with different concentrations of FX5 $(2,5$, or $10 \mu \mathrm{M})$ for $24 \mathrm{~h}$, and $0.5 \mathrm{mg} / \mathrm{mL} \mathrm{MTT}$ was added into the medium for additional $4 \mathrm{~h}$. After incubation, $100 \mu \mathrm{L}$ DMSO was added to dissolve formazan crystals, and absorbance at $490 \mathrm{~nm}$ was measured using a Spectra Max i3x reader (Molecular Devices, Sunnyvale, CA, USA).

Animals

All animal experiments were performed following the institutional ethical guidelines on animal care of Nanjing University of Chinese Medicine. All mice were maintained under standard conditions at room temperature $\left(22^{\circ} \mathrm{C}\right.$ ) by a $12 \mathrm{~h}$ light/dark cycle. Four (for establishing T2DM model) and 8 (for establishing T1DM model)week-old C57BL/6 male mice were purchased from Vital River Laboratory Animal Technology Co (Beijing, China).

T1DM mice. 8-week-old C57BL/6 male mice were fed for 7 days to acclimatize and then received a single i.p. injection of $150 \mathrm{mg} /$ $\mathrm{kg} \mathrm{STZ}$ in $0.5 \mathrm{M}$ sodium citrate buffer or vehicle. One week after injection, mice with blood glucose level higher than $16.7 \mathrm{mM}$ were classified as T1DM (STZ) mice [34]. 
T2DM mice. 4-week-old C57BL/6 male mice were fed for 7 days to acclimatize and then divided into normal diet group and high-fat diet (HFD) group. The normal diet group mice were fed with a standard laboratory diet and the HFD group mice were fed with a formulation comprising $60 \mathrm{kcal} \%$ fat for 4 weeks to induce insulin resistance. All mice were then fasted for $6 \mathrm{~h}$ and the HFD group

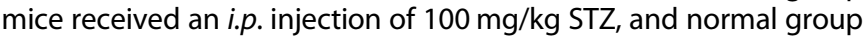
mice received the same volume of vehicle. T2DM (HFD/STZ) mice were identified as those with blood glucose level higher than 11.1 $\mathrm{mM}$ after 1 week [35].

AAV-induced GR knockdown mice. AAV-ePHP-si-GR (AAV-si-GR) [31] and negative control vector (AAV-si-NC) were purchased from Shanghai Genechem Co (Shanghai, China). The titer of AAV-si-GR was $3.14 \times 10^{13} \mu \mathrm{g} / \mathrm{mL}$. AAV-induced GR knockdown diabetic mice $(\mathrm{STZ}+\mathrm{AAV}-\mathrm{si}-\mathrm{GR}$; HFD/STZ + AAV-si-GR) were obtained via tail vein injection with a dose of $1 \times 10^{11} \mu \mathrm{g} /$ mouse [36] 2 weeks after diabetic model was established.

The knockdown efficiency of AAV injection was detected 2 weeks after diabetic model was established (Supplementary Fig. S1a-d).

\section{Animal administration}

Diabetic mice were randomized into diabetic group and FX5 (20, 40 $\mathrm{mg} / \mathrm{kg}$ ) group. Four weeks after diabetic model was established, the animals occurred cognitive impairment as reported [37]. Diabetic mice in FX5 groups were administered FX5 by gastric perfusion, and mice in control ( $\mathrm{C} 57 \mathrm{BL} / 6$ male mice) and model group were given the same amount of vehicle (2\% DMSO, $5 \%$ Tween- 80 ).

AAV-injected diabetic mice were randomized into AAV-si-NC group, AAV-si-GR group, and AAV-si-GR + FX5 (40 mg/kg) group. After 2 weeks, FX5 and vehicle were administered by gastric perfusion.

Mice administrations were performed for 8 weeks, once a day. $n$ $=25$ for T1DM/group, and $n=15$ for T2DM/group.

Open field test

The open field test (OFT) is a straightforward test to evaluate the locomotion and exploratory behavior in mice [38]. Before OFT testing, mice were transferred to the testing room and acclimated for at least $1 \mathrm{~h}$. Mice were then tested in a clear plastic chamber $(32 \mathrm{~cm} \times$ $32 \mathrm{~cm} \times 20 \mathrm{~cm}$ ) for $15 \mathrm{~min}$. The apparatus was cleaned with $70 \%$ alcohol after testing of each mouse. Total movement in the open field and the time spent in center were recorded for data analysis.

\section{Novel object recognition test}

Novel object recognition (NOR) test is based on rodent's natural curiosity to explore the immediate environment [39]. The test consists of four sequential daily trials. During the habituation trial, mice were placed in the center of the open-field apparatus and allowed to explore freely for $15 \mathrm{~min}$. During the acquisition trial, each animal was given $5 \mathrm{~min}$ to freely explore two identical objects (Object A) and then returned to the home cage. After $4 \mathrm{~h}$, mice were returned to the test arena where one of the sample objects had been replaced by a novel one (Object B). The apparatus and objects were cleaned with $70 \%$ ethanol before use and between each animal test. All data were collected for animal performance analysis. During the test phase, a discrimination index was calculated.

\section{Y-maze test}

As described previously [40], the Y-maze $(46 \mathrm{~cm} \times 11 \mathrm{~cm} \times 25 \mathrm{~cm})$ consists of three arms (A, B and C) at $120^{\circ}$ angles to each other. Before testing, mice were allowed to habit in the testing room for $30 \mathrm{~min}$. For the training trials, one arm of the maze was blocked off (novel arm, N). The mice were placed in the intersection of three arms and allowed to move freely in the other two arms (other arm, O) for $5 \mathrm{~min}$. Two hours later, the blocked arm was opened, and the mice were allowed to explore again in the three arms for $5 \mathrm{~min}$. A mouse was considered to have entered an arm when all four paws were positioned in the arm runway. Total arm entries were measured and the percentage number of entries into $\mathrm{N}$ was calculated as follows: $\% \mathrm{~N}=(100 \% \times \mathrm{N}) /(\mathrm{N}+\mathrm{O})$.

Morris water maze test

Morris water maze (MWM) test was performed according to the published approach [41]. Briefly, for training stage, mice were trained to find an invisible submerged platform placed in the circular pool $(120 \mathrm{~cm}$ in diameter, $50 \mathrm{~cm}$ deep) filled with white food additives. Mice were given $60 \mathrm{~s}$ to find the platform and allowed to stay at the platform for $10 \mathrm{~s}$. If a mouse failed to find the platform within $60 \mathrm{~s}$, it was pulled gently to the platform and kept there for $10 \mathrm{~s}$. For testing stage, the platform was removed. Mice were given $60 \mathrm{~s}$ to search for the platform. All data were collected for animal performance analysis. In data analysis, the pool was artificially divided into four equal quadrants formed by imaging lines, intersected in the center of the pool at right angles by north, south, east, and west.

\section{Golgi staining}

Morphology of dendritic spines in the brain was analyzed using a FD Rapid Golgi Stain Kit (FD Neuro technologies, Elliot City, MD, USA) by manufacturer's instructions. Images for neurons were obtained with an automated upright microscope (Leica Microsystems, Nussloch, Germany).

\section{Electrophysiology test}

Long-term potentiation (LTP) assay was performed by published approach [31]. In the assay, the baseline was recorded for $10 \mathrm{~min}$, and the LTP in the DG area was then induced by four trains of 100 $\mathrm{Hz}$ stimuli with the same intensity of the test stimulus. The strength of synaptic transmission was determined by measuring the initial (20\%-80\% rising phase) slope of field excitatory postsynaptic potentials (fEPSPs).

Immunoblot assay

The assay was conducted based on the published method [42].

Cell-based assay. Primary neurons were incubated with PA (0.2 $\mathrm{mM})$ or/and different concentrations of FX5 $(2,5$, or $10 \mu \mathrm{M})$ for 24 $\mathrm{h}$ and primary microglia were incubated with LPS $(100 \mathrm{ng} / \mathrm{mL})$ or/ and different concentrations of FX5 $(2,5$, or $10 \mu \mathrm{M})$ for $24 \mathrm{~h}$, and then lysed with RIPA buffer containing protease inhibitor cocktail and phosphatase inhibitor cocktail on ice for $20 \mathrm{~min}$, followed by centrifugation at $12,000 \mathrm{r} / \mathrm{min}$ for $30 \mathrm{~min}$ at $4^{\circ} \mathrm{C}$.

Brain tissue-based assay. Brain tissues of mice in each group were homogenized with RIPA buffer containing protease inhibitor cocktail and phosphatase inhibitor cocktail by a hand-hold motor and kept on ice for $1 \mathrm{~h}$. After the tissues were lysed completely, the homogenates were centrifuged at $12,000 \mathrm{r} / \mathrm{min}$ for $30 \mathrm{~min}$ at $4{ }^{\circ} \mathrm{C}$.

The supernatants were collected, and protein concentrations were determined using BCA protein assay kit. Proteins were mixed with $2 \times$ SDS-PAGE sample buffer and then boiled for $15 \mathrm{~min}$ at $95^{\circ} \mathrm{C}$.

Cell or tissue extracts were separated by SDS-PAGE and transferred to a nitrocellulose filter membrane (GE Healthcare, Waukesha, WI, USA). After blocking for $2 \mathrm{~h}$ at room temperature, the membranes were incubated with corresponding antibodies (dilution 1:1000) overnight at $4{ }^{\circ} \mathrm{C}$. The membranes were washed and incubated with secondary anti-rabbit/mouse antibodies (dilution 1:3000) conjugated with horseradish peroxidase for $2 \mathrm{~h}$ at room temperature. The blots were developed and visualized using a ChemiDoc MP (Bio-Rad, Hercules, CA, USA).

The details of antibodies were provided in Supplementary Table S1.

RNA isolation and quantitative real-time PCR

Total RNA in cells and brain tissues was extracted by RNAiso plus regent according to the manufacturer's protocol [43], and $1 \mu \mathrm{g}$ total RNA was reverse-transcribed into cDNA using Prime Script RT 
reagent kit (Takara Biomedical Technology Co, Beijing, China). Finally, mRNA levels of different genes were quantified by quantitative real-time PCR using SYRB Premix Ex Taq kit and normalized to GAPDH or $\beta$-Actin. The thermal cycling condition was $95^{\circ} \mathrm{C}$ for $10 \mathrm{~s}$ followed by 40 cycles of amplification at $95^{\circ} \mathrm{C}$ for $10 \mathrm{~s}, 60^{\circ} \mathrm{C}$ for $20 \mathrm{~s}, 72^{\circ} \mathrm{C}$ for $10 \mathrm{~s}$ and $80^{\circ} \mathrm{C}$ for $1 \mathrm{~s}$, then keeping at $72{ }^{\circ} \mathrm{C}$ for $10 \mathrm{~min}$.

The primers used in real-time PCR were shown in Supplementary Table S2.

\section{SiRNA plasmid transfection}

Primary neurons or primary microglia were transfected with $50 \mathrm{nM}$ si-NC or si-GR. si-NC or si-GR was dissolved in $25 \mu \mathrm{L}$ of Opti-MEM and mixed with Lipofectamine 2000 transfection reagent for 15 min, then cells were incubated with the mixture. After $6 \mathrm{~h}$, the medium was changed into DMEM or DMEM/F12 and incubated for $24 \mathrm{~h}$. si-NC was used as a negative control.

\section{Immunostaining}

Synaptic integrity analysis. Primary neurons were incubated with PA $(0.2 \mathrm{mM})$ or/and different concentrations of FX5 $(2,5$, or $10 \mu \mathrm{M})$ for $24 \mathrm{~h}$, then primary neurons grown on slides were washed three times with PBS and fixed in $4 \%$ paraformaldehyde for $5 \mathrm{~min}$ at room temperature. The cells were then incubated with $0.3 \%$ Triton X-100 for $10 \mathrm{~min}$ and blocked with $4 \%$ BSA for $30 \mathrm{~min}$ at room temperature, followed by incubation with VAMP2 or PSD95 antibodies (dilution 1:500) overnight at $4{ }^{\circ} \mathrm{C}$. The slide was washed three times and incubated with fluorescent secondary detection antibodies (dilution 1:250) goat anti-rabbit and goat anti-mouse for $1 \mathrm{~h}$ and the nucleus was stained with Hoechst 33342.

Brain tissues. The left hemisphere was fixed in 4\% paraformaldehyde overnight and kept in $30 \%$ sucrose until sank to the bottom. The tissues were embedded in OCT and sectioned by using a cryostat microtome. For detecting the expression of GR, MAP2, Iba1, NF-KB and NLRP3, the slides were incubated in 5\% Triton $X-100$ for $10 \mathrm{~min}$ and blocked in $5 \%$ BSA for $30 \mathrm{~min}$ at room temperature, followed by incubation overnight at $4{ }^{\circ} \mathrm{C}$ with primary antibody (dilution 1:400). The slides were then washed three times and incubated with fluorescent secondary detection antibodies (dilution 1:250) for $1 \mathrm{~h}$ and the nucleus was stained with Hoechst 33342.

The antibodies used for immunostaining were provided in Supplementary Table S3. Images were acquired using a Leica fluorescence microscope and analyzed by ImageJ software.

\section{DAB staining}

Brain slides $(20 \mu \mathrm{m})$ were incubated in $5 \%$ Triton X-100 for 10 min and blocked in 5\% BSA for $30 \mathrm{~min}$ at room temperature, followed by incubation with AT8 antibody (Invitrogen, St, Louis, MO, USA) (dilution 1:250) overnight at $4{ }^{\circ} \mathrm{C}$. Then the slides were washed three times and incubated with anti-mice secondary antibody for $1 \mathrm{~h}$ and subsequently with diaminobenzidine for $5 \mathrm{~min}$ and washed three times [44]. Images were obtained with an automated upright microscope.

\section{Nissl staining}

The slides were washed twice and incubated in Cresyl violet Stain for $30 \mathrm{~min}$ at $37^{\circ} \mathrm{C}$. Slides were washed with $\mathrm{dd}_{2} \mathrm{O}$ for $5 \mathrm{~s}$ and placed in Nissl differentiation for several seconds to 2 min until the background of the slides was close to achromatic. Then the slides were dehydrated in ethanol for $5 \mathrm{~s}$. Finally, xylene solution was dropped to the slides sealed with neutral gum [45]. Images were obtained with an automated upright microscope.

Statistical analysis

Data were presented as mean \pm SEM. Unpaired two-tailed Student's $t$ test was used for two-group comparison. One-way
ANOVA with Dunnett's post-test was used for at least three group's comparisons. Significance was defined as $P<0.05$.

\section{RESULTS}

FX5 ameliorated synaptic impairment through GR/BDNF/TrkB/ CREB pathway in PA-treated primary neurons

FX5 was determined as a non-steroidal GR antagonist by mammalian one-hybrid and transactivation assays as well as nuclear translocation assay in our previous work [30]. Notably, the antagonistic activity of FX5 against other nuclear receptors was also evaluated by mammalian transactivation assay. The results indicated that the antagonism of FX5 against GR (60\%) was obviously superior to that of MR (27\%), PR (27\%), LXR $(28 \%)$ and ERß (29\%) (Supplementary Fig. S1e-i).

Here, we investigated the potential of FX5 in ameliorating $\mathrm{DCl}$. Since MTT assay result demonstrated that FX5 rendered no influence on primary neuron viability at 2,5 and $10 \mu \mathrm{M}$ (Supplementary Fig. S1j), these three concentrations were set in the following experiments.

PA is a common fatty acid in human diet and the plasma PA level is elevated in diabetic patients [46]. PA is widely used to establish AD-like model by inducing tau hyperphosphorylation [47], apoptosis [48], and ER stress [46]. Moreover, increased tissue PA concentrations may also increase GCs concentrations [49]. In our study, immunostaining results demonstrated that PA increased GR expression and promoted GR nuclear translocation in U2OS/GR-GFP cells, which strengthened the feasibility of PA for establishing $\mathrm{DCl}$ model in vitro. In addition, FX5 antagonized PA-induced GR expression and GR nuclear translocation (Supplementary Fig. S1k-m).

FX5 protected synaptic integrity by antagonizing GR. Given that synaptic loss is responsible for $\mathrm{DCl}$ [50], immunostaining and immunoblot assays were performed to inspect the potential of FX5 in protecting synaptic integrity by detecting synaptic integrity-related proteins PSD95, synaptophysin (SYN) and VAMP2 in primary neurons. As shown in Fig. $1 \mathrm{~b}-\mathrm{e}, \mathrm{FX} 5$ antagonized PAinduced suppression of these proteins. Moreover, si-GR deprived FX5 of its above capability (Fig. 1f, g). All results demonstrated that FX5 protected synaptic integrity by antagonizing GR in PA-treated primary neurons.

FX5 upregulated BDNF/TrkB/CREB pathway by antagonizing $G R$. Given that BDNF/TrkB/CREB signaling functions potently in the regulation of synapse plasticity [51], we inspected the potential regulation of FX5 against this pathway in PA-treated primary neurons. Immunoblot results demonstrated that FX5 antagonized PA-induced repression of the protein levels of BDNF, $p$-TrkB and p-CREB (Fig. $1 \mathrm{~h}, \mathrm{i}$ ), and si-GR deprived FX5 of its antagonistic ability (Fig. 1j,k). These results indicated that FX5 upregulated BDNF/ TrkB/CREB pathway by antagonizing GR.

Thus, all results demonstrated that FX5 ameliorated synaptic impairment through GR/BDNF/TrkB/CREB pathway in PA-treated primary neurons.

FX5 protected against neuronal apoptosis by repressing GR/PI3K/ AKT/GSK3 $\beta$ pathway-mediated tauopathy and subsequent endoplasmic reticulum (ER) stress in PA-treated primary neurons FX5 protected against neuronal apoptosis by antagonizing GR. Brain nerve injury and neuronal apoptosis are important pathological changes of $\mathrm{DCl}$ [52]. We performed immunoblot assay and the results indicated that FX5 antagonized the PAinduced downregulation of anti-apoptotic proteins (Bcl-xl and $\mathrm{Bcl}-$ 2) and upregulation of pro-apoptotic proteins (cleaved caspase-3 (C-C3) and Bax) (Fig. 1l, m), while si-GR deprived FX5 of its antiapoptotic capability (Fig. 1n, o) in PA-treated primary neurons. All results implied that FX5 protected against neuronal apoptosis by antagonizing GR. 
a<smiles>O=S(=O)(Nc1ccc(Cl)c(C(F)(F)F)c1)c1ccc(Cl)s1</smiles>

d

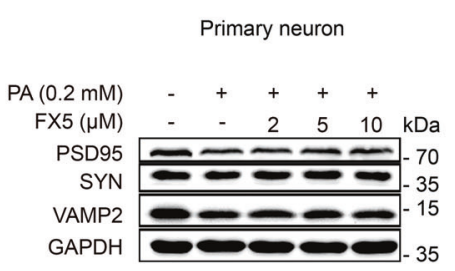

h

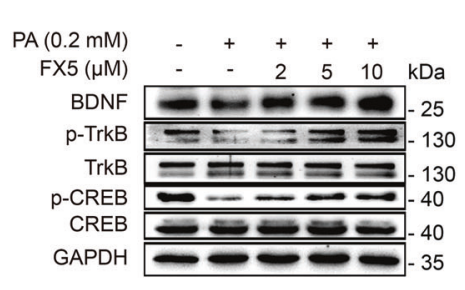

I

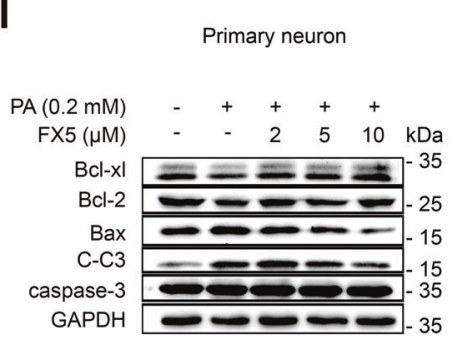

p

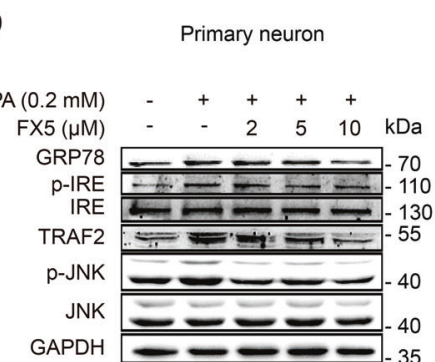

b

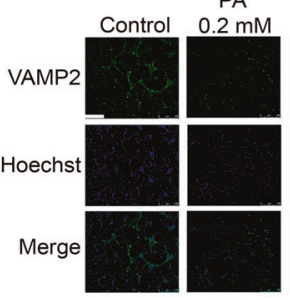

e

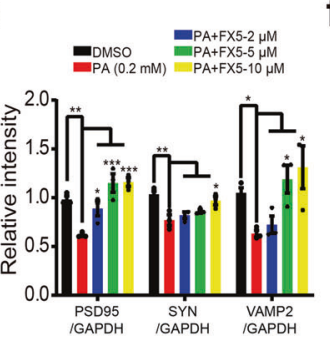

i

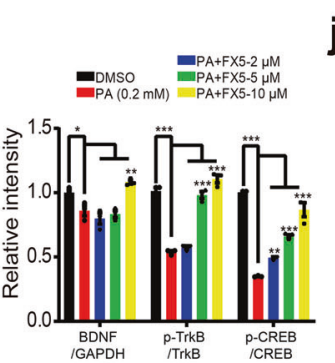

j



Primary neuron

$\frac{\text { Si-NC }}{+++} \frac{\text { Si-GR }}{-++}$

Primary neuron

si-NC si-GR

$\begin{array}{lllllll}\mathrm{PA}(0.2 \mathrm{mM}) & - & + & + & - & + & + \\ \mathrm{FX} 5(10 \mu \mathrm{M}) & - & - & + & - & - & +\end{array}$

PSD95 $-m-7-70$

SYN

VAMP2 $2-1-\sqrt{-35}$

m

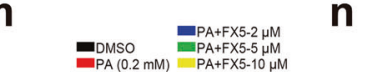

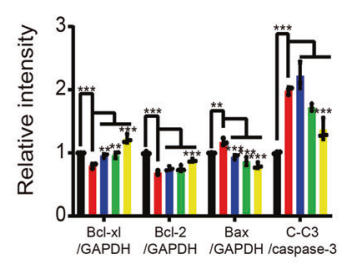

q

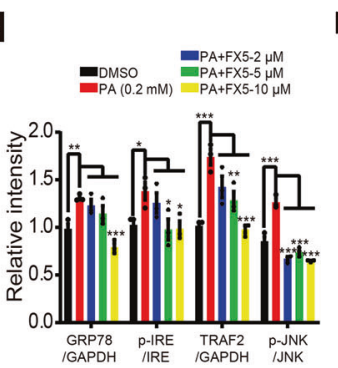

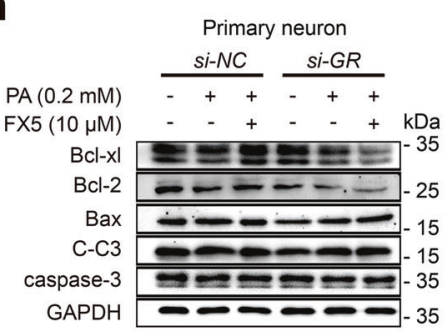

$\mathbf{r}$

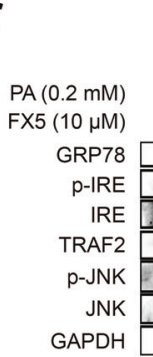

Primary neuron

Si-NC Si-GR

$-++\ldots++$

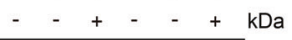

$-70$

$-110$

$\div-\cdots-130$

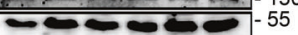

- -40

- $=-40$ k

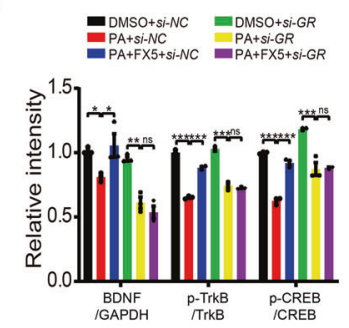

0
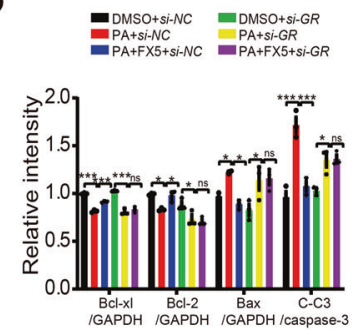

$\mathbf{s}$
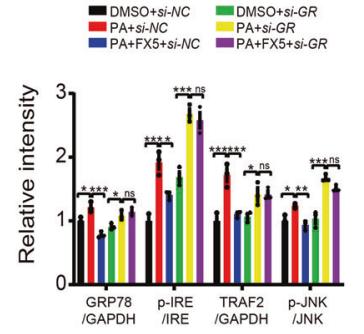

Fig. 1 FX5 ameliorated synaptic impairment and protected against neuronal apoptosis and ER stress in PA-treated primary neurons. (a) Chemical structure of FX5. Immunostaining assays for synapse-related proteins (b) VAMP2 (Scale bar: $250 \mu \mathrm{m})$ and (c) PSD95 (Scale bar: $50 \mu \mathrm{m})$ in PA-treated primary neurons $(n=4)$. Immunoblot analysis with quantifications showed that $(\mathbf{d}$, e) FX5 antagonized the PA-induced suppression of synapse-related proteins (PSD95, SYN, and VAMP2) and (f, $\mathbf{g})$ si-GR deprived FX5 of its antagonistic capability in PA-treated primary neurons $(n=3)$. Immunoblot analysis with quantifications showed that (h, i) FX5 antagonized PA-induced repression on the protein levels of BDNF, p-TrkB and p-CREB and $(\mathbf{j}, \mathbf{k})$ si-GR deprived FX5 of its antagonistic capability in PA-treated primary neurons $(n=3)$. Immunoblot analysis with quantifications showed that (I, m) FX5 antagonized PA-induced decrease in anti-apoptotic proteins (Bcl-2 and Bcl-xl) and increase in pro-apoptotic proteins (Bax and C-C3), and $(\mathbf{n}, \mathbf{o})$ si-GR deprived FX5 of its antagonistic capability in PA-treated primary neurons $(n=3)$. Immunoblot analysis with quantifications showed that $(\mathbf{p}, \mathbf{q})$ FX5 inhibited PA-induced ER stress and $(\mathbf{r}, \mathbf{s})$ si-GR deprived FX5 of its inhibitory capability in PA-treated primary neurons $(n=3)$. GAPDH was used as loading control. $n=3$ independent experiments. Mean \pm SEM. One-way ANOVA followed by Dunnett's multiple comparison test. * $P<0.05$, ${ }^{* *} P<0.01,{ }^{* * *} P<0.001$ vs PA group. 
FX5 repressed ER stress by antagonizing GR. ER stress was tightly implicated in cognitive impairment [53] and accumulated unfolded proteins in ER promoted dissociation of ER chaperone GRP78 from IRE, causing IRE interaction with TRAF2 and further activation of downstream target JNK ultimately resulting in apoptosis [54]. Immunoblot assay was next performed in PAtreated primary neurons. The results demonstrated that FX5 inhibited PA-induced ER stress by antagonizing the upregulation of protein levels of GRP78, p-IRE, TRAF2, and p-JNK (Fig. 1p, q). Moreover, si-GR deprived FX5 of its above-mentioned antagonistic capabilities (Fig. 1r,s), implying that FX5 repressed ER stress by targeting GR.

FX5 attenuated tau hyperphosphorylation by antagonizing GR. Considering that tau hyperphosphorylation is highly contributable to neurofibrillary tangles formation in brain with DCl [55], and accumulation of the hyperphosphorylated tau as a misfolded protein in the ER could cause neurodegeneration due to protracted ER stress [56, 57], immunoblot assay was detected and the results demonstrated that FX5 antagonized PA-stimulated tau hyperphosphorylation at Ser199, Thr231 and Ser396 in primary neurons (Fig. 2a, b). Notably, si-GR deprived FX5 of its antagonistic capability against tau hyperphosphorylation (Fig. 2c, d). These results thus demonstrated that FX5 attenuated tau hyperphosphorylation by antagonizing GR.

FX5 regulated PI3K/AKT/GSK3 $\beta$ pathway by antagonizing GR. Since activated PI3K induces AKT activation to phosphorylate varied substrates including GSK3 $\beta$, and PI3K/AKT signaling dysfunction activates GSK3 $\beta$ leading to tau hyperphosphorylation [8], we examined the regulation of FX5 against the protein levels of p-PI3K, p-AKT (Ser 473) and p-GSK3 3 (Ser 9) in PA-treated primary neurons. Immunoblot results indicated that FX5 antagonized PA-induced decline in these proteins (Fig. 2e, f), and si-GR deprived FX5 of its antagonistic capability against $\mathrm{PI} 3 \mathrm{~K} / \mathrm{AKT} / \mathrm{GSK} 3 \beta$ pathway (Fig. $2 \mathrm{~g}, \mathrm{~h}$ ). Thus, these results demonstrated that FX5 regulated $\mathrm{PI3K} / \mathrm{AKT} / \mathrm{GSK} 3 \beta$ pathway by antagonizing GR.

Together, all results demonstrated that FX5 protected against neuronal apoptosis by repressing GR/PI3K/AKT/GSK3 $\beta$ pathway-mediated tauopathy and subsequent ER stress in PAtreated primary neurons.

FX5 repressed inflammation through GR/NF-KB/NLRP3/ASC/ Caspase-1 pathway in LPS-treated primary microglia

FX5 repressed inflammation by antagonizing GR. As inflammation contributes highly to DCI [58], we detected the potential inhibitory capability of FX5 against inflammation in LPS-treated primary microglia by RT-PCR. The results indicated that FX5 antagonized LPS-induced upregulation of pro-inflammatory factors TNF-a and IL-1 $\beta$ (Fig. 2i, j), and si-GR deprived FX5 of its antagonistic capability to these pro-inflammatory factors (Fig. $2 \mathrm{k}, \mathrm{l}$ ). These results thus implied that FX5 repressed inflammation by antagonizing GR.

FX5 repressed NF-KB/NLRP3/ASC/Caspase-1 pathway by antagonizing GR. NF-KB and NLRP3 are tightly implicated in inflammation [59], and NLRP3 inflammasome becomes activated when exposed to stimuli, in that NLRP3 undergoes a conformational change and assembles with adaptor protein ASC and proCaspase 1 resulting in activation of Caspase 1 and further formation of mature IL-1 $\beta$ [60]. With these facts, immunoblot assay was performed in primary microglia and the results indicated that FX5 antagonized LPS-induced upregulation of $\mathrm{p}$ NF-KB, NLRP3, ASC and Caspase-1 (P20) (Fig. 2m,n), and si-GR deprived FX5 of its antagonistic capability against NLRP3 inflammasome (Fig. 2o, p).
Given the potent regulation of NF-KB against NLRP3 inflammasome [59], we further investigated whether NF-KB signaling was required for NLRP3 regulation by using NF-KB inhibitor PDTC in the assay [61]. As shown in Fig. 2q-s, PDTC blocked the capability of FX5 in antagonizing LPS-induced upregulation of protein and mRNA levels of NLRP3 in primary microglia. These results implied that FX5 repressed NF-KB/ NLRP3/ASC/Caspase-1 pathway by antagonizing GR.

Therefore, all results demonstrated that $\mathrm{FX} 5$ repressed inflammation through GR/NF-KB/NLRP3/ASC/Caspase-1 pathway in LPS-treated primary microglia.

FX5 ameliorated $\mathrm{DCl}$ in mice by antagonizing GR

To further investigate the capability of FX5 in ameliorating $\mathrm{DCl}$, the cognitive ability of diabetic mice was evaluated by OFT, NOR, Y-maze, and MWM tests with FX5 $(20,40 \mathrm{mg} / \mathrm{kg})$ treatment. Moreover, to confirm that FX5 ameliorated $\mathrm{DCl}$ in mice by antagonizing GR, the related assays were performed in AAV-si-GRinjected diabetic mice with brain GR-specific knockdown (Fig. 3a, d).

OFT test. OFT test was used to investigate the autonomous and exploratory behaviors of mice in a new environment [62]. As indicated in Supplementary Fig. S2a-d, FX5-treated (STZ + FX5; HFD/ $\mathrm{STZ}+\mathrm{FX} 5$ ) or AAV-si-GR-injected (STZ + AAV-si-GR; HFD/STZ + AAV$s i-G R)$ diabetic mice spent more time in the center compared with vehicle-treated (STZ; HFD/STZ) diabetic mice. In addition, no significant difference was found in the totally moved distance among the mice in all groups (Supplementary Fig. S2e-h).

NOR test. This test was to evaluate the short-term working memory of mice [63]. As indicated in Fig. 3e-h, FX5 or AAV-si-GR treatment increased the discrimination index of diabetic mice.

$Y$-maze test. This test was to examine spatial working memory of mice [64]. As expected, the percentage of number in novel arms of FX5 or AAV-si-GR-treated diabetic mice was increased compared with that in vehicle-treated diabetic mice (Fig. $3 \mathrm{i}-\mathrm{I}$ ).

In addition, the result that there was no difference in total arm entries of T2DM mice in all groups (Supplementary Fig. S2j and I) indicated no deficit in motor function of T2DM mice, while FX5 or AAV-si-GR treatment failed to ameliorate the total arm entries in T1DM mice (Supplementary Fig. S2i and k). These results implied that the motor ability of T1DM mice was more severely impaired than that of T2DM.

MWM test. MWM test was typically to examine spatial learning and long-term memory of mice [65]. As indicated in Fig. 3m, o, q and s, FX5 or AAV-si-GR-treated diabetic mice spent shorter time in finding the hidden platforms in terms of daily escape latency through 5-day (for STZ mice) or 4-day (for HFD/STZ mice) consecutive training compared with vehicle-treated diabetic mice. The different training days for diabetic mice reflected that the cognitive impairment of T1DM is more serious than that of T2DM. Testing trails were conducted after the training and the results demonstrated that FX5 or AAV-si-GR-treated diabetic mice crossed the target quadrant more frequently than vehicle-treated diabetic mice (Fig. 3n, p, $r$ and $t$ ).

Notably, AAV-si-GR injection deprived FX5 of its abovementioned therapeutic capability against the corresponding indicators of OFT test (Supplementary Fig. S2c-d), NOR test (Fig. 3g, h), Y-maze test (Fig. 3k, I) and MWM test (Fig. 3q, t) in AAVsi-GR injected diabetic mice.

Collectively, all results demonstrated that FX5 ameliorated DCI by antagonizing GR, and such amelioration had no impacts on the levels of blood glucose, body weight and diet in diabetic mice (Supplementary Fig. S2m-x). 

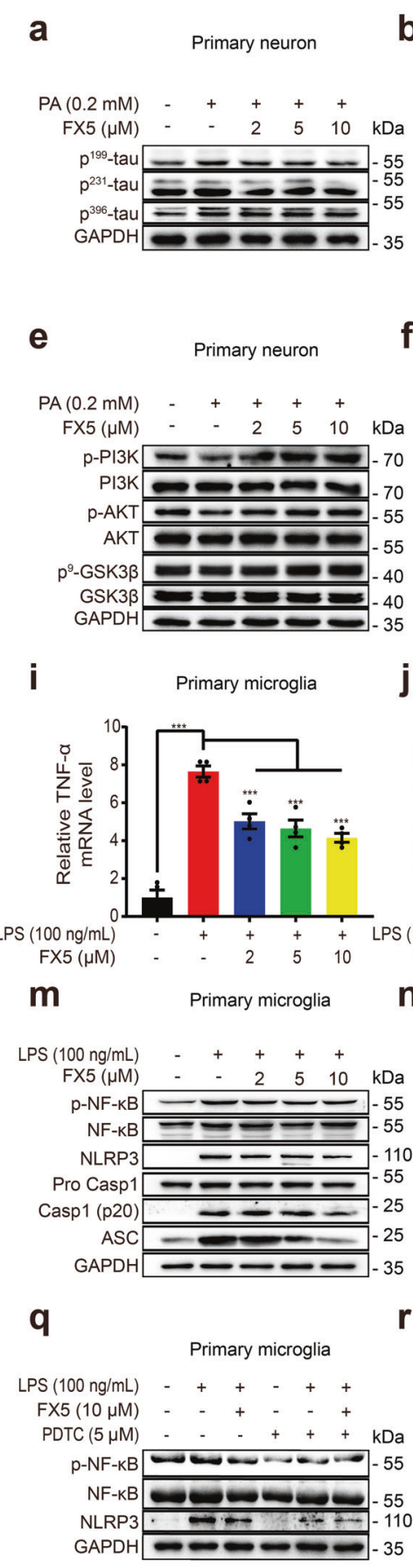

b

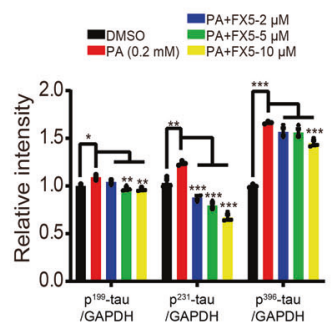

f

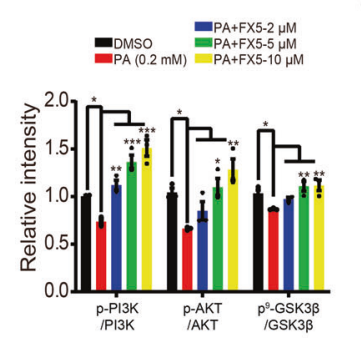

C

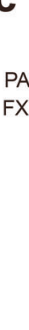

g

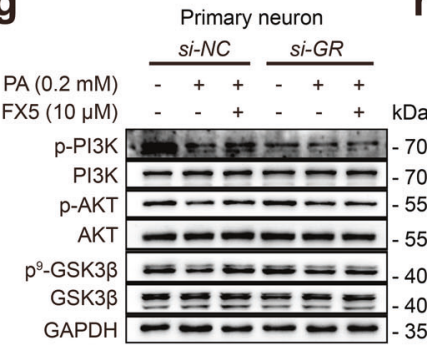

k

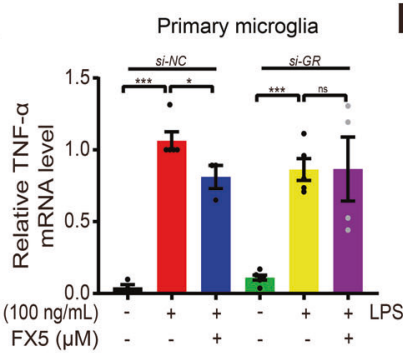

0

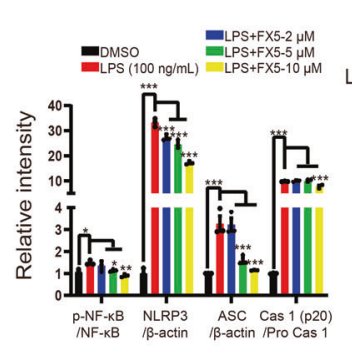

$\mathrm{PA}(0.2 \mathrm{mM}) \frac{\text { si-NC }}{-++} \frac{\text { Si-GR }}{-++}$

$\mathrm{F} 5(10 \mu \mathrm{M})--+-\quad+\mathrm{kDa}$

$\mathrm{p}^{231}$-tau $\longrightarrow-55$

$\mathrm{p}^{396} \mathrm{tau}=\equiv$

GAPDH $\longrightarrow-35$

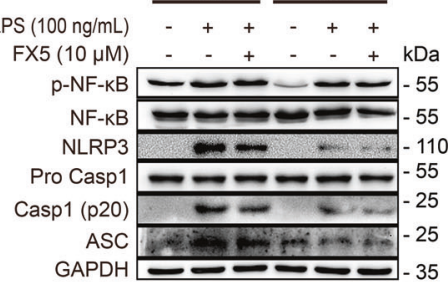

$\mathrm{p}^{199-\mathrm{tau}}=-55$

h

d
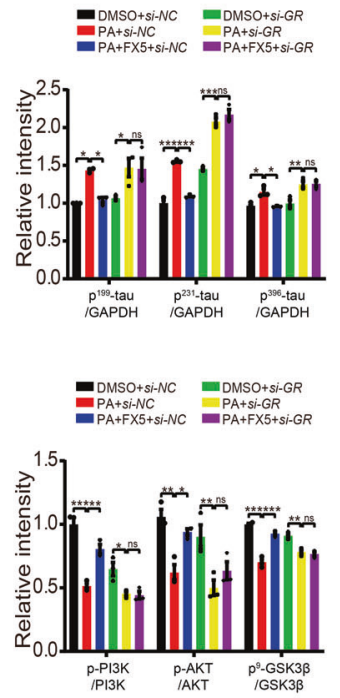

I Primary microglia

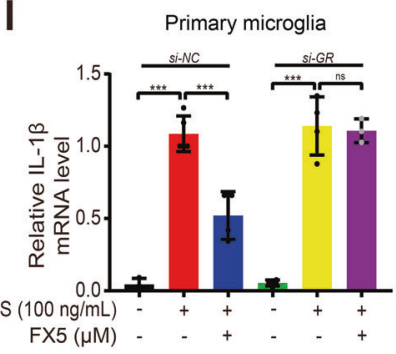

p

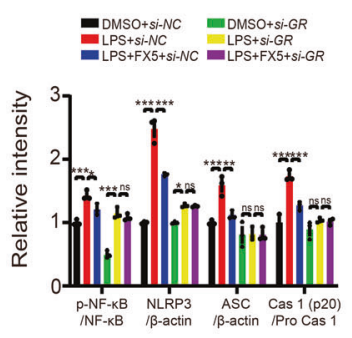

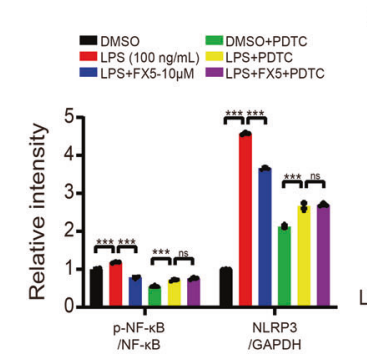

S

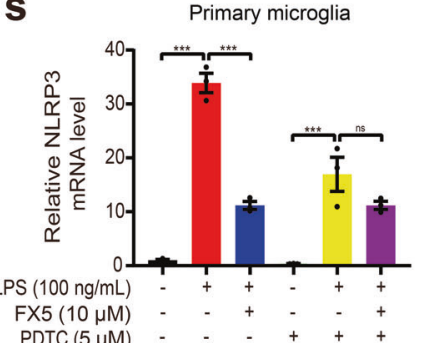

Fig. 2 FX5 attenuated tauopathy in PA-treated primary neurons and repressed inflammation in LPS-treated primary microglia. Immunoblot analysis with quantifications showed that (a, b) FX5 antagonized PA-stimulated tau hyperphosphorylation at Ser199, Thr231 and Ser396 and (c, d) si-GR deprived FX5 of its antagonistic capability in PA-treated primary neurons $(n=3)$. Immunoblot analysis with quantifications showed that (e, f) FX5 antagonized PA-induced decline in phosphorylation levels of PI3K, AKT (Ser 473) and GSK3 $\beta$ (Ser 9) and $(\mathbf{g}, \mathbf{h})$ si-GR deprived FX5 of its antagonistic capability in PA-treated primary neurons $(n=3)$. RT-PCR results demonstrated that $(\mathbf{i}, \mathbf{j})$ FX5 antagonized LPS-stimulated expression levels of pro-inflammatory factors TNF- $\alpha$ and IL-1 $\beta$, and (k, I) si-GR deprived FX5 of its antagonistic capability in LPS-treated primary microglia $(n=4)$. Immunoblot analysis with quantifications showed that $(\mathbf{m}, \mathbf{n})$ FX5 antagonized LPS-induced upregulation of p-NF-kB, NLRP3, ASC and Caspase-1 (P20), and (o, p) si-GR deprived FX5 of its antagonistic capability in LPS-treated primary microglia $(n=3)$. (q) Immunoblot analysis with $(\mathbf{r})$ quantifications and (s) RT-PCR result showed that treatment of NF-KB inhibitor PDTC abolished FX5-induced suppression against NLRP3 in LPS-treated primary microglia $(n=3)$. GAPDH was used as loading control. $n=3$ independent experiments. Mean \pm SEM. One-way ANOVA followed by Dunnett's multiple comparison test. ${ }^{*} P<0.05,{ }^{* *} P<0.01,{ }^{* * * P} P<0.001$ vs PA or LPS group. 
a

T1DM mice

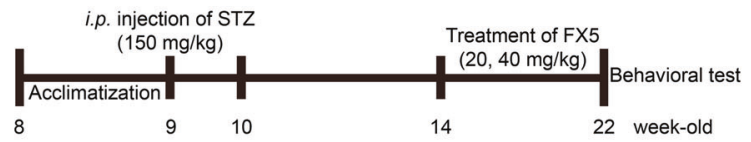

C

AAV-injected T1DM mice

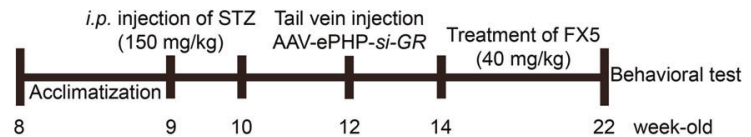

e

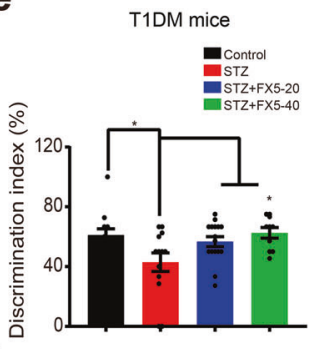

i

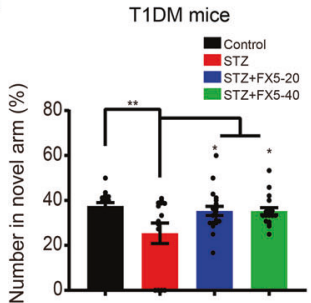

m

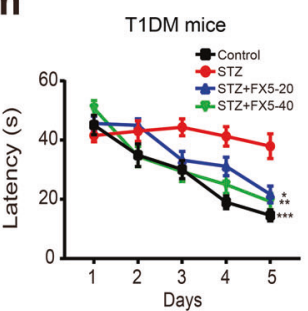

$\mathbf{q}$

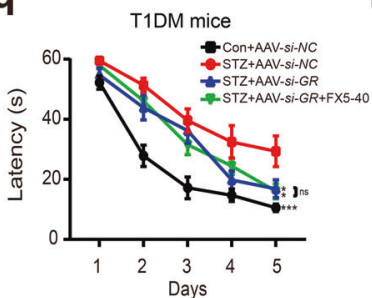

f

f T2DM mice

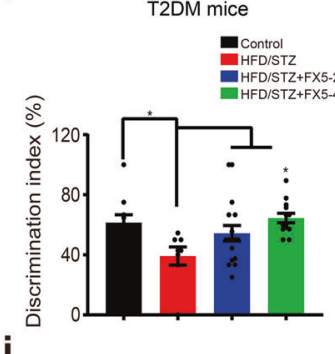

j

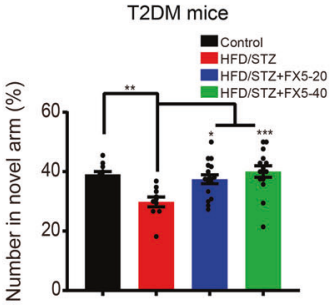

n
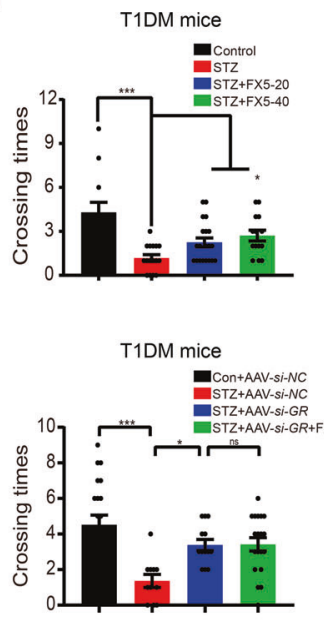

b

T2DM mice

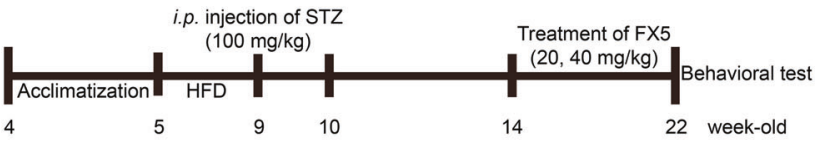

d

AAV-injected T2DM mice

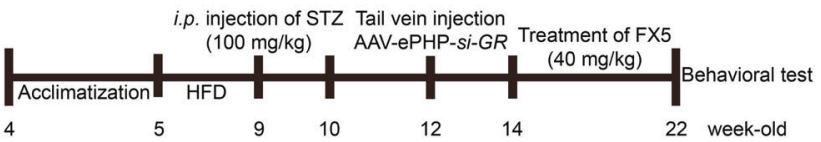

g

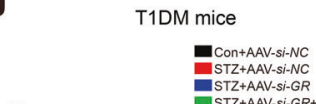

h

T2DM mice

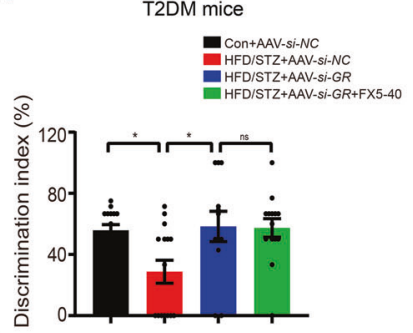

k
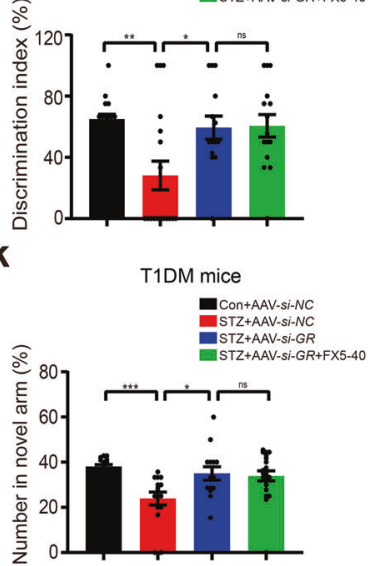

I

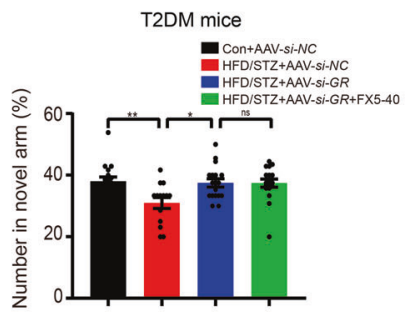

o

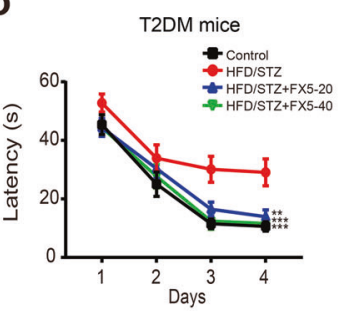

p

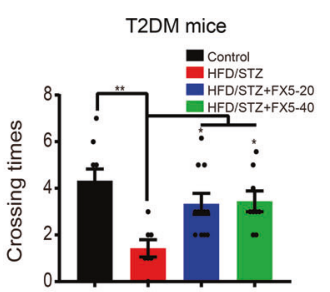

s
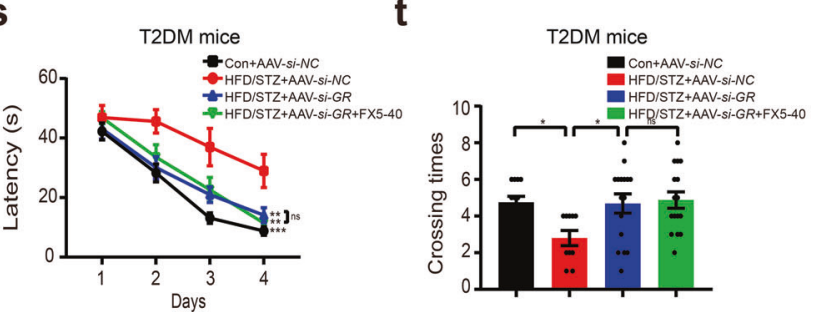

Fig. 3 FX5 ameliorated DCI in mice by antagonizing GR. Schedules of animal treatment and behavior test to (a, c) STZ and (b, d) HFD/STZ mice. NOR test results indicated that FX5 $(20,40 \mathrm{mg} / \mathrm{kg})$ or AAV-si-GR treatment ameliorated short-term working memory defects in (e, g) STZ and (f, h) HFD/STZ mice ( $n \geq 8$ per group), and FX5 $(40 \mathrm{mg} / \mathrm{kg}$ ) treatment had no impacts on such defects in (g) STZ + AAV-si-GR and (h) HFD/ STZ + AAV-si-GR mice ( $n \geq 12$ per group). Y-maze test results indicated that FX5 $(20,40 \mathrm{mg} / \mathrm{kg})$ or AAV-si-GR treatment ameliorated spatial working memory defects in (i, k) STZ and (j, I) HFD/STZ mice ( $n \geq 10$ per group), and FX5 (40 mg/kg) treatment had no impacts on such defects in (k) STZ + AAV-si-GR and (I) HFD/STZ + AAV-si-GR mice ( $n \geq 14$ per group). Escape latency results during platform trials indicated that FX5 (20, $40 \mathrm{mg} / \mathrm{kg}$ ) or AAV-si-GR treatment ameliorated learning and memory dysfunction in (m, q) STZ and (o, s) HFD/STZ mice ( $n \geq 7$ per group), and FX5 $(40 \mathrm{mg} / \mathrm{kg})$ treatment had no impacts on such dysfunctions in (q) STZ + AAV-si-GR and (s) HFD/STZ + AAV-si-GR mice ( $n \geq 12$ per group). Times of platform crossing in probe trials for (n) STZ, (p) HFD/STZ, (r) STZ + AAV-si-NC and (t) HFD/STZ + AAV-si-NC mice ( $n \geq 7$ per group). Mean \pm SEM. One-way ANOVA followed by Dunnett's multiple comparison test. ${ }^{*} P<0.05$, ${ }^{* *} P<0.01$, ${ }^{* * *} P<0.001$ vs model or AAV-si-NC treated model group. 
FX5 ameliorated synaptic impairment through GR/BDNF/TrkB/ CREB pathway in diabetic mice

Since we have determined the protection of FX5 against synaptic impairment in primary neurons, we next evaluated such a protective effect in mice. Considering that we have determined the regulation of FX5 against GR nuclear translocation in U2OS/ GR-GFP cells, we firstly investigated whether FX5 also exhibited such an effect in mice. For this purpose, neurons and microglia in the hippocampus of diabetic mice were identified by MAP2 and Iba1 antibodies [31] respectively, and immunostaining results indicated that FX5 inhibited GR nuclear translocation in brain neurons and microglia of diabetic mice (Supplementary Fig. S3a-h).

FX5 protected synaptic integrity by antagonizing GR. Golgi staining assay was applied to evaluate the development of dendrites and the number of dendritic spines of neurons. As shown in Fig. $4 a$ and b, FX5 or AAV-si-GR treatment ameliorated synaptic integrity deficiency in the hippocampus of diabetic mice.

In addition, FX5 or AAV-si-GR treatment upregulated the levels of synaptic integrity-related proteins PSD95, SYN, and VAMP2 in the hippocampus and cortex of diabetic mice (Fig. 4c-j and Supplementary Fig. S3i-p), and AAV-si-GR injection deprived FX5 of its capability in upregulating the levels of these proteins in AAVsi-GR-treated diabetic mice (Fig. $4 a-j$ and Supplementary Fig. S3i-p). These results demonstrated that FX5 improved synapse integrity in diabetic mice by antagonizing GR.

FX5 ameliorated LTP by antagonizing GR. Given that LTP of synaptic transmission is commonly applied to detect synaptic plasticity [31], LTP in hippocampal DG region was examined. As indicated in Fig. 4k-n, FX5 or AAV-si-GR treatment improved LTP induction and maintenance in diabetic mice, and AAV-si-GR injection deprived FX5 of its capability in improving synaptic transmission in AAV-si-GR-treated diabetic mice. Thus, all results demonstrated that FX5 improved synapse plasticity in diabetic mice by antagonizing GR.

FX5 upregulated BDNF/TrkB/CREB pathway by antagonizing GR. Next, we examined the regulation of FX5 treatment against the protein levels of BDNF, $p$-TrkB and $p$-CREB in the hippocampus and cortex of diabetic mice like the case in cell-based assays (Fig. $1 \mathrm{~h}, \mathrm{i}$ ). As shown in Fig. 4o-v and Supplementary Fig. S3q-X, FX5 or AAV-si-GR treatment upregulated the levels of these proteins in diabetic mice, and AAV-si-GR deprived FX5 of its capability in upregulating these proteins in AAV-si-GR-treated diabetic mice. Thus, all results demonstrated that FX5 treatment upregulated BDNF/TrkB/CREB pathway in diabetic mice by antagonizing GR.

Together, all results demonstrated that FX5 ameliorated synaptic impairment through GR/BDNF/TrkB/CREB pathway in diabetic mice.

FX5 protected against neuronal apoptosis by repressing GR/PI3K/ AKT/GSK3 $\beta$ pathway-mediated tauopathy and subsequent ER stress in diabetic mice

FX5 suppressed neuronal apoptosis by antagonizing GR. To evaluate the protective effect of FX5 on neurons in mice, we performed Nissl staining and immunoblot assays. The results indicated that the number of survival neurons in $\mathrm{CA} 1$ region and the levels of anti-apoptotic proteins $\mathrm{Bcl}-2$ and $\mathrm{BCl}-\mathrm{xl}$ were increased, while the levels of pro-apoptotic proteins Bax and C-C3 were decreased in the hippocampus and cortex of FX5 or AAV-si-GR-treated diabetic mice compared with those in vehicletreated diabetic mice, indicative of the capability of FX5 treatment in suppressing neuronal apoptosis. Notably, AAV-si-GR treatment deprived FX5 of its above-mentioned capability in AAV-si-GRtreated diabetic mice (Fig. 5a-I and Supplementary Fig. S4a-h).
These results demonstrated that FX5 suppressed neuronal apoptosis in diabetic mice by antagonizing GR.

FX5 repressed ER stress by antagonizing GR. Next, immunoblot assay was performed to investigate whether FX5 treatment repressed ER stress by antagonizing GR in the hippocampus and cortex of diabetic mice. As shown in Fig. $5 \mathrm{~m}-\mathrm{t}$ and Supplementary Fig. S4i-p, FX5 or AAV-si-GR treatment suppressed the levels of ER stress-related proteins GRP78, p-IRE, TRAF2 and p-JNK in diabetic mice. Notably, AAV-si-GR injection deprived FX5 of its suppressive capability in AAV-si-GR-treated diabetic mice. All results demonstrated that FX5 repressed ER stress in diabetic mice by antagonizing GR.

FX5 attenuated tauopathy by antagonizing GR. We next detected the ability of FX5 in attenuating tauopathy by immunoblot assays and DAB staining in the hippocampus and cortex of diabetic mice. As indicated in Fig. 6a-p and Supplementary Fig. S5a-p, FX5 or AAV-si-GR treatment decreased the levels of tau phospho-epitopes and AT8 protein, and AAV-si-GR treatment deprived FX5 of its above-mentioned capability in AAV-si-GR-treated diabetic mice. Therefore, all results demonstrated that FX5 attenuated tauopathy in diabetic mice by antagonizing GR.

FX5 regulated PI3K/AKT/GSK3 $\beta$ pathway by antagonizing GR. Like the case in cell-based assay, we examined the levels of p-PI3K, p-AKT (Ser 473) and p-GSK3 $\beta$ (Ser 9) in the hippocampus and cortex of diabetic mice. Immunoblot results suggested that FX5 or AAV-si-GR treatment upregulated these phosphorylated proteins in diabetic mice, and AAV-si-GR treatment deprived FX5 of its such capability in AAV-si-GR-treated diabetic mice (Fig. $6 \mathrm{q}-\mathrm{x}$ and Supplementary Fig. $S 5 q-x)$. These results demonstrated that FX5 regulated PI3K/AKT/GSK3 $\beta$ pathway by antagonizing GR in diabetic mice.

Together, FX5 protected against neuronal apoptosis by repressing GR/PI3K/AKT/GSK3 $\beta$ pathway-mediated tauopathy and subsequent ER stress in diabetic mice.

FX5 repressed inflammation through GR/NF-KB/NLRP3/ASC/ Caspase-1 pathway in diabetic mice

FX5 treatment ameliorated inflammation by antagonizing GR. Like the case in cell-based assay, the levels of inflammatory factors TNF- $\alpha$ and IL-1 $\beta$ in the hippocampus and cortex of diabetic mice were detected by RT-PCR and immunoblot assays. As shown in Fig. $7 \mathrm{a}-\mathrm{h}, \mathrm{m}-\mathrm{t}$ and Supplementary Fig. S6a-p, FX5 or AAV-si-GR treatment suppressed the levels of these inflammatory factors in diabetic mice, and AAV-si-GR treatment deprived FX5 of its suppressive effect in AAV-si-GR-injected diabetic mice. These results thus demonstrated that FX5 ameliorated inflammation by antagonizing GR.

FX5 repressed microglial NLRP3 inflammasome by antagonizing GR. As FX5 was capable of repressing activation of NLRP3 inflammasome by antagonizing $G R$ in primary microglia (Fig. $2 m-p)$, we next inspected such a capability of FX5 in the hippocampus of diabetic mice. In the assay, microglia were identified by Iba1 antibody. Immunostaining results demonstrated that FX5 or AAV-si-GR treatment suppressed NLRP3 positive rate in diabetic mice and AAV-si-GR injection deprived FX5 of its suppressive capability in AAV-si-GR- injected diabetic mice (Fig. 7i, j and Supplementary Fig. S6q, r).

FX5 suppressed microglial NF-KB nuclear translocation by antagonizing $G R$. Given the tight implication of NF-KB in inflammation, we inspected the potential of FX5 in regulating NF-KB nuclear translocation by immunostaining assay in microglia of the hippocampus of diabetic mice. As shown in Fig. $7 \mathrm{k}, \mathrm{I}$ and Supplementary Fig. S6s, t, FX5 or AAV-si-GR treatment inhibited 


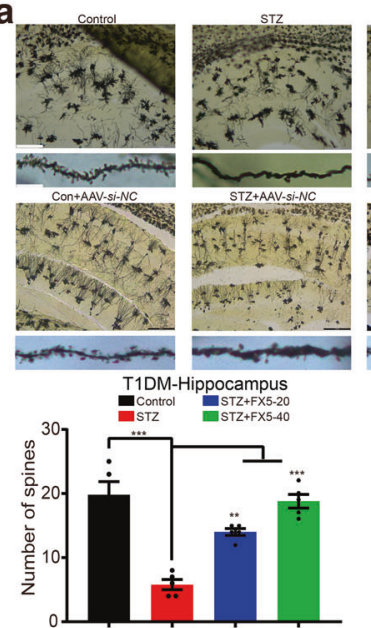

C

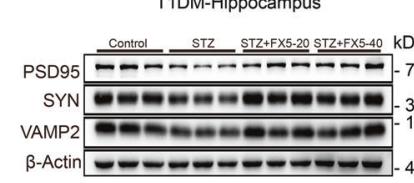

g
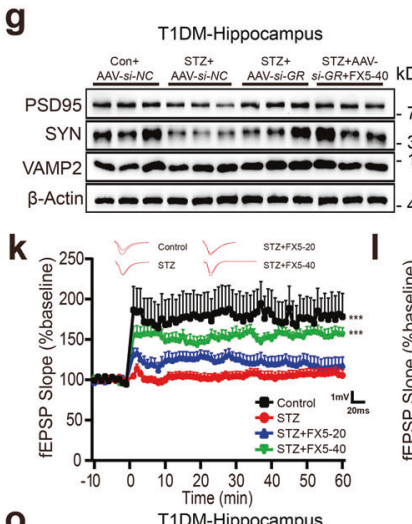

o
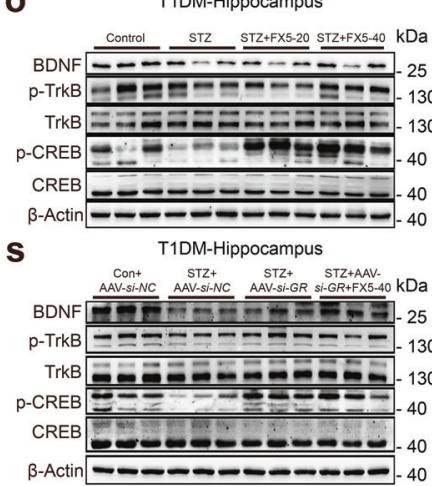

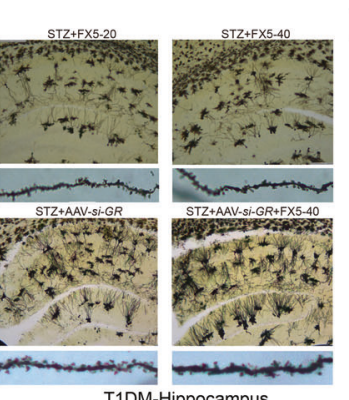

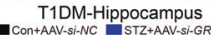

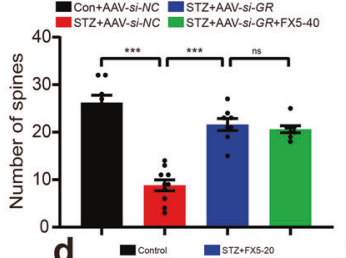

d
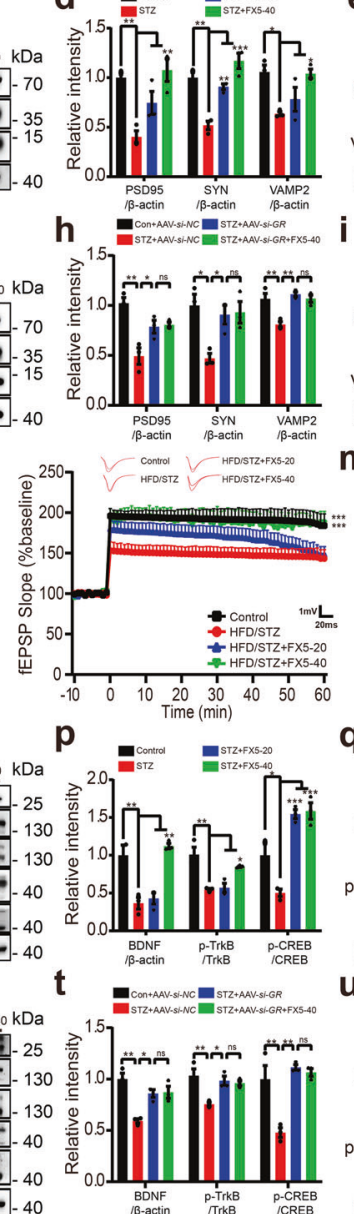

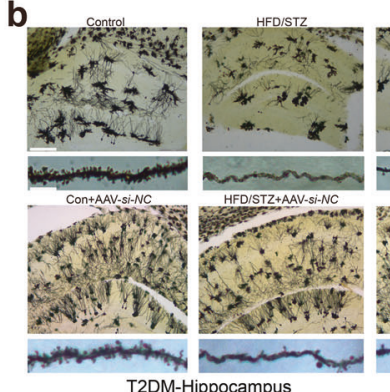

T2DM-Hippocampus
EControl E HFD/STZ+FX5-20
EFD/STZ HFD/STZ+FX5-40

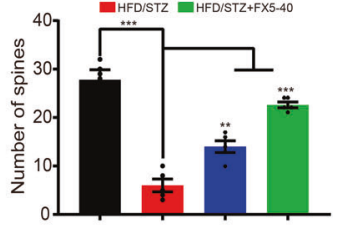

e
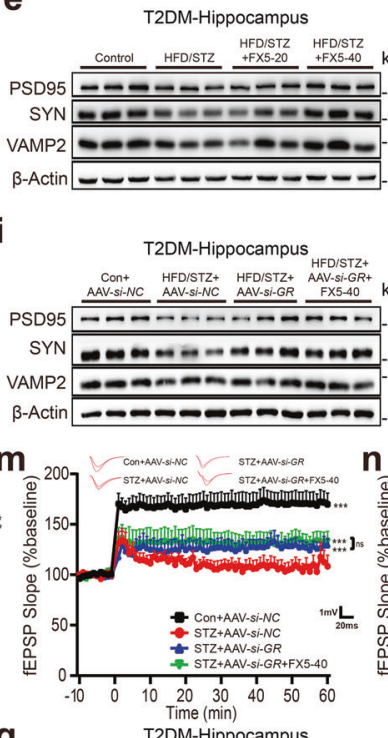

q

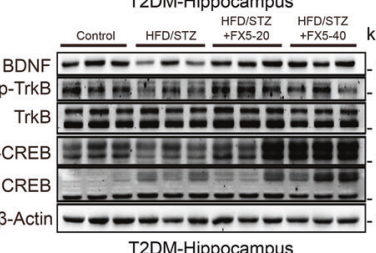

$\mathbf{u}$ T2DM-Hippocampus Cont HFD/STZ+ HFDIT/ HFD/STZ+

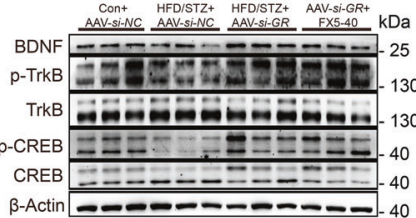

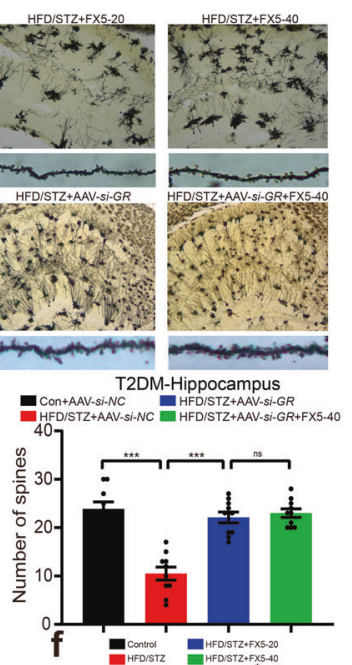
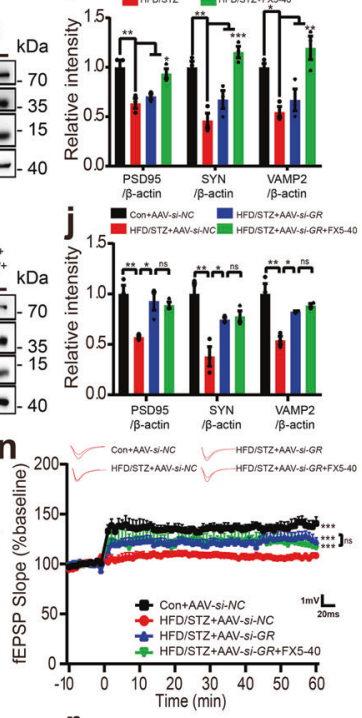
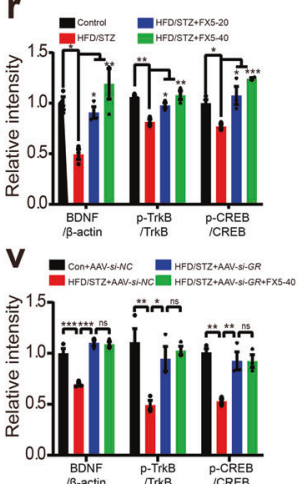

Fig. 4 FX5 ameliorated synaptic impairment through GR/BDNF/TrkB/CREB pathway in the hippocampus of diabetic mice. FX5 (20, 40 mg/ $\mathrm{kg}$ ) or AAV-si-GR treatment reversed the spine density deficiency of hippocampal neuron in (a) STZ and (b) HFD/STZ mice, and FX5 (40 mg/kg) had no impacts on such a deficiency in STZ + AAV-si-GR and HFD/STZ + AAV-si-GR mice ( $n \geq 5$ per group). Scale bar: $200 \mu \mathrm{m}, 10 \mu \mathrm{m}$. FX5 ( 20 , $40 \mathrm{mg} / \mathrm{kg}$ ) or AAV-si-GR treatment increased the expression of PSD95, SYN and VAMP2 in the hippocampus of (c, d, g, h) STZ and (e, f, i, j) HFD/ STZ mice, and FX5 $(40 \mathrm{mg} / \mathrm{kg})$ rendered no influence on these proteins in $(\mathbf{g}, \mathbf{h}) \mathrm{STZ}+\mathrm{AAV}-\mathrm{si}-\mathrm{GR}$ and $(\mathbf{i}, \mathbf{j}) \mathrm{HFD} / \mathrm{STZ}+\mathrm{AAV}$-si-GR mice $(n=3 \mathrm{per}$ group). Changes in fEPSP slope were recorded following high frequency stimulation $(4 \times 100 \mathrm{~Hz})$, and FX5 (20, $40 \mathrm{mg} / \mathrm{kg})$ or AAV-si-GR treatment ameliorated LTP impairments in the hippocampal DG region of $(\mathbf{k}, \mathbf{m})$ STZ and (I, $\mathbf{n}) \mathrm{HFD} / \mathrm{STZ}$ mice, while FX5 (40 mg/kg) had no impacts on LTP impairments in (m) STZ + AAV-si-GR and (n) HFD/STZ + AAV-si-GR mice (animal, $n=3$ per group; brain slice, $n \geq 6$ per group). FX5 $(20,40 \mathrm{mg} / \mathrm{kg})$ or AAV-si-GR treatment upregulated the expressions of BDNF, p-TrkB and p-CREB in the hippocampus of (o, p, s, t) STZ and $(\mathbf{q}, \mathbf{r}, \mathbf{u}, \mathbf{v}) \mathrm{HFD} / \mathrm{STZ}$ mice, and FX5 $(40 \mathrm{mg} / \mathrm{kg})$ had no impacts on these proteins in (s, t) STZ + AAV-si-GR and (u, v) HFD/STZ + AAV-si-GR mice $(n=3$ per group). $\beta$-Actin was used as loading control. $n=3$ independent experiments. Mean \pm SEM. One-way ANOVA followed by Dunnett's multiple comparison test. ${ }^{*} P<0.05,{ }^{* *} P<0.01,{ }^{* *} P<0.001$ vs model or AAV-si-NC treated model group. 
a

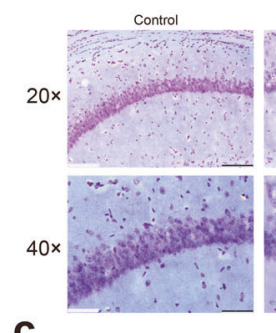

C

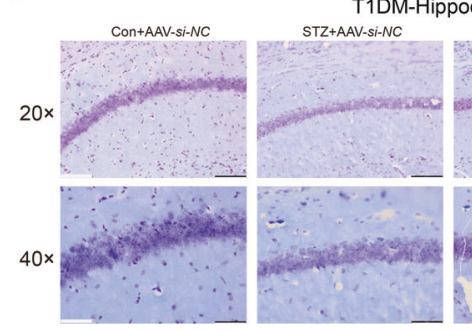

e

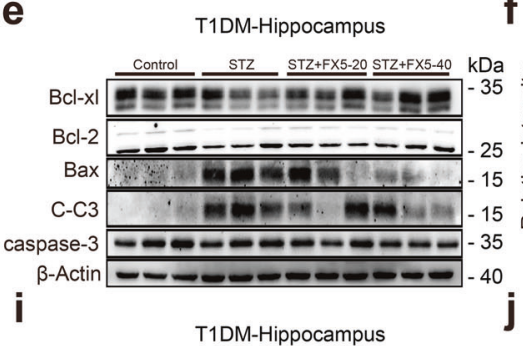$$
\text { i }
$$

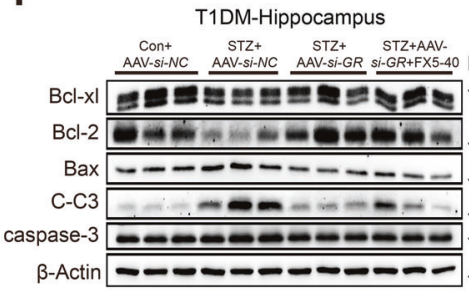

m

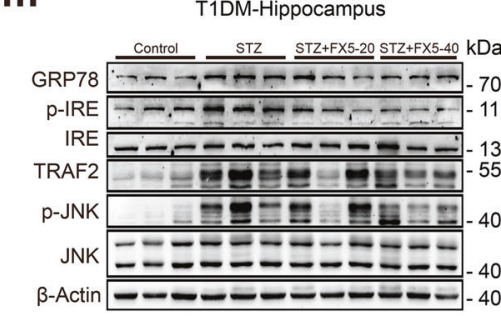

q

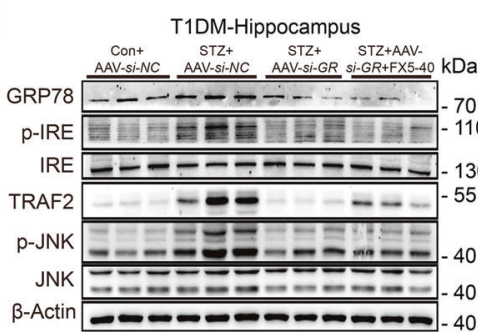

j
T1DM-Hippocampus

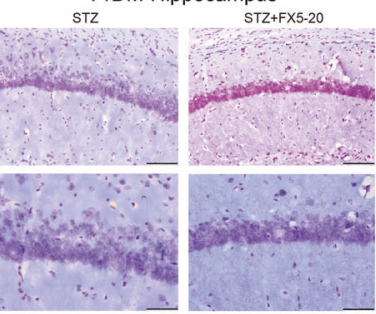

pocampus

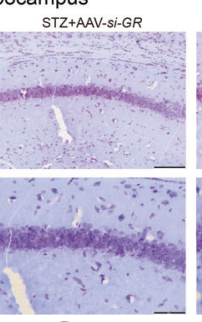

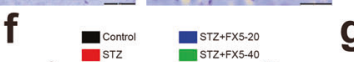

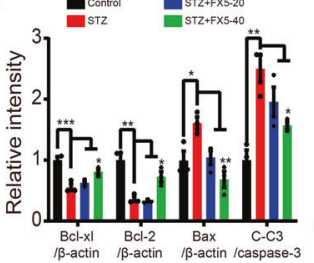

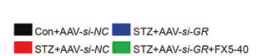



n

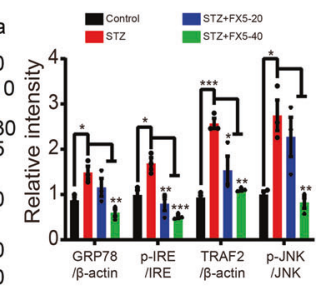

$r \quad s$

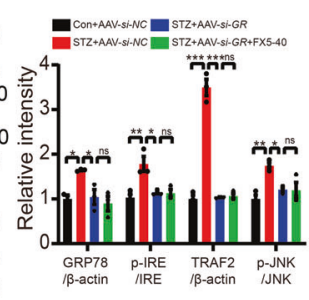

b

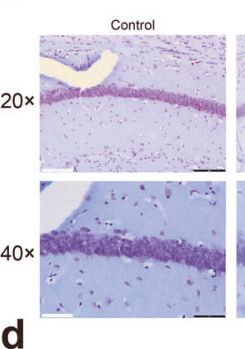

d

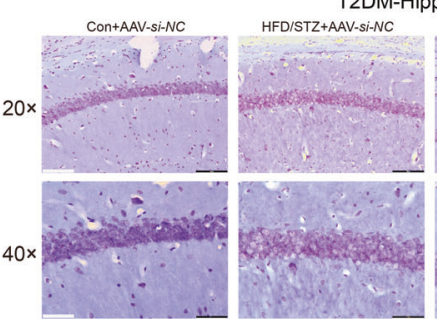

g

T2DM-Hippocampus

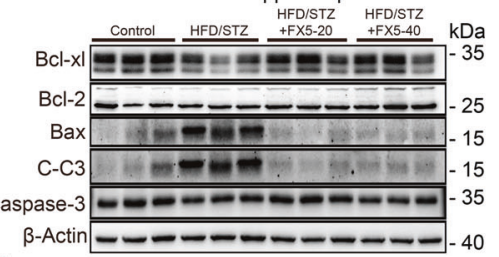

k

T2DM-Hippocampus HFDSTZ+ $_{\text {ST }}$

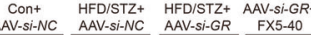

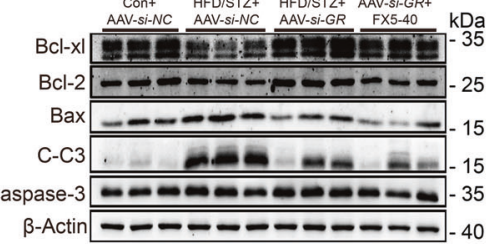

O

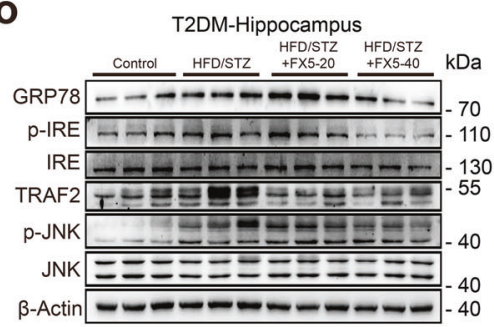

$\mathbf{s}$

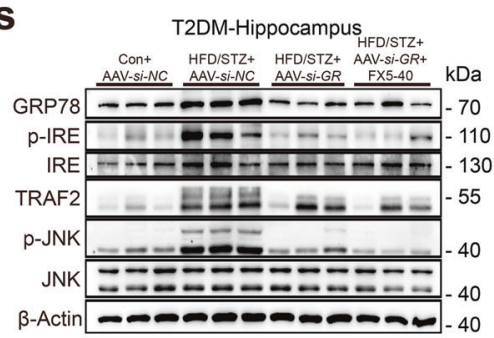

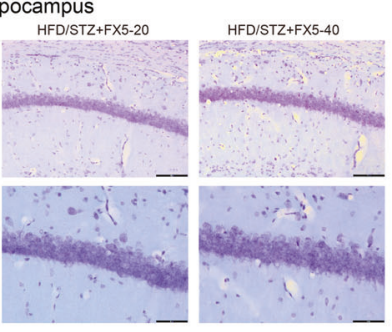

pocampus

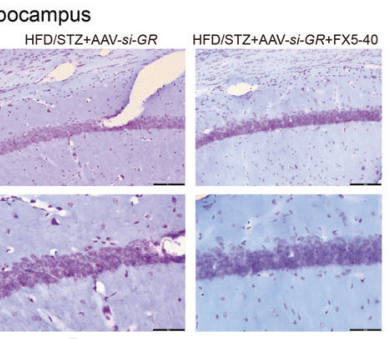

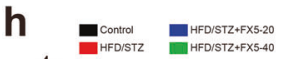
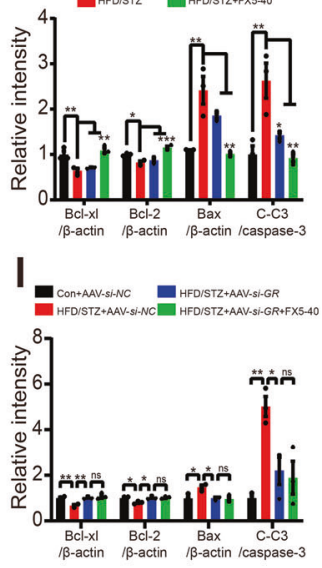

p

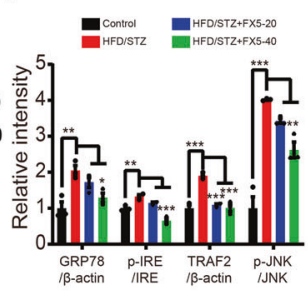

$\mathbf{t}$

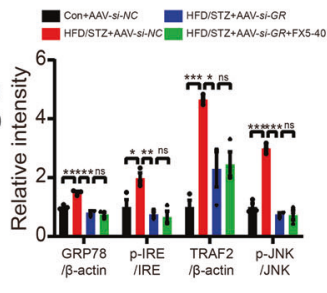

Fig. 5 FX5 protected against neuronal apoptosis and ER stress in the hippocampus of diabetic mice. Nissl staining results indicated that FX5 $(20,40 \mathrm{mg} / \mathrm{kg})$ or AAV-si-GR treatment increased the number of survival neurons in the hippocampus of (a, c) STZ and (b, d) HFD/STZ mice, and FX5 $(40 \mathrm{mg} / \mathrm{kg})$ treatment had no such an effect in (c) STZ + AAV-si-GR and (d) HFD/STZ + AAV-si-GR mice ( $n=4$ per group). Scale bar: $100 \mu \mathrm{m}, 50 \mu \mathrm{m}$. Immunoblot analysis with quantifications showed that FX5 $(20,40 \mathrm{mg} / \mathrm{kg})$ or AAV-si-GR treatment suppressed proapoptotic process in the hippocampus of $(\mathbf{e}, \mathbf{f}, \mathbf{i}, \mathbf{j})$ STZ and $(\mathbf{g}, \mathbf{h}, \mathbf{k}, \mathbf{l}) \mathrm{HFD} / \mathrm{STZ}$ mice, and FX5 (40 mg/kg) treatment had no such effects in (i, j) STZ + AAV-si-GR and (k, I) HFD/STZ + AAV-si-GR mice ( $n=3$ per group). Immunoblot analysis with quantifications showed that FX5 (20, 40 mg/ $\mathrm{kg}$ ) or AAV-si-GR treatment ameliorated ER stress in the hippocampus of ( $\mathbf{m}, \mathbf{n}, \mathbf{q}, \mathbf{r})$ STZ and (o, p, s, t) HFD/STZ mice, and FX5 (40 mg/kg) treatment had no such an ameliorative effect in $(\mathbf{q}, \mathbf{r})$ STZ + AAV-si-GR and (s, t) HFD/STZ + AAV-si-GR mice $(n=3$ per group). $\beta$-Actin was used as loading control. $n=3$ independent experiments. Mean \pm SEM. One-way ANOVA followed by Dunnett's multiple comparison test. ${ }^{*} P<0.05$, ${ }^{* *} P<0.01,{ }^{* *} P<0.001$ vs model or AAV-si-NC treated model group. 
a
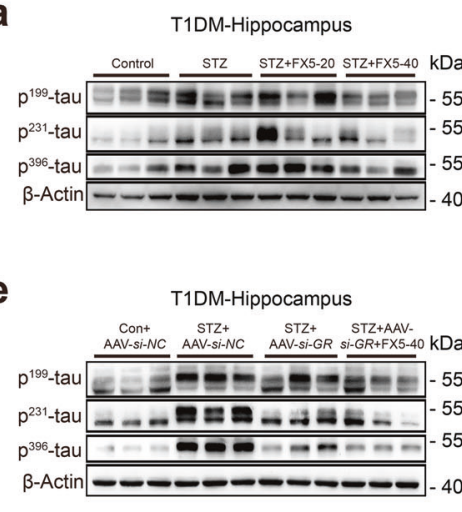

i
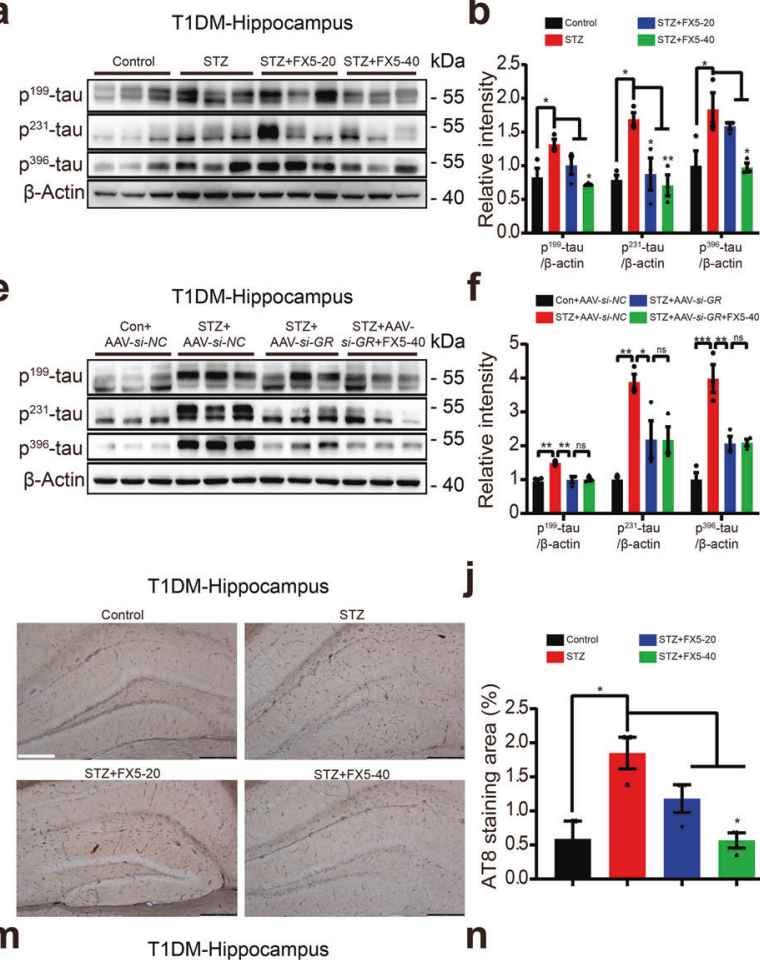

f

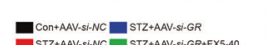
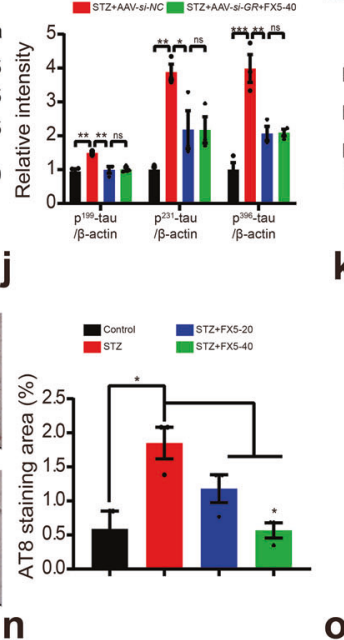

C

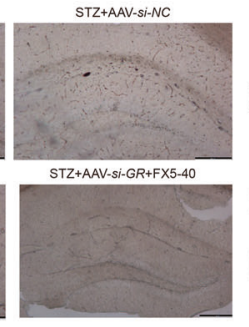

$\mathbf{q}$

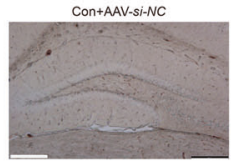

STZ+AAV-Si-GR

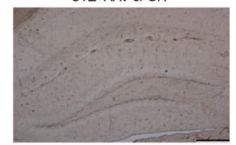

T1DM-Hippocampus

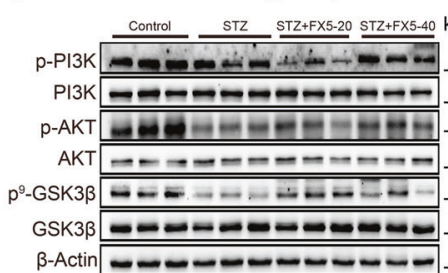

$\mathbf{u}$

T1DM-Hippocampus
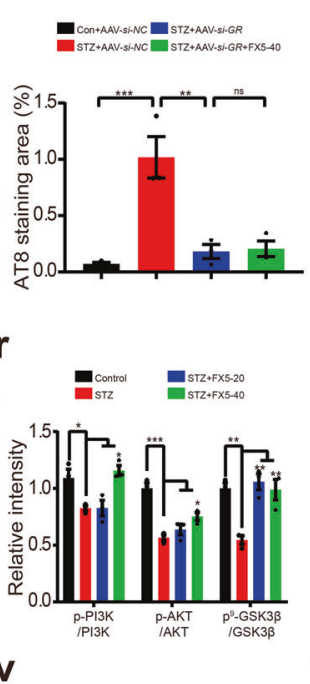

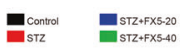

V

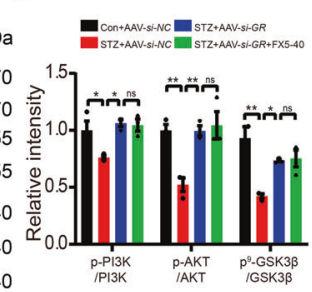

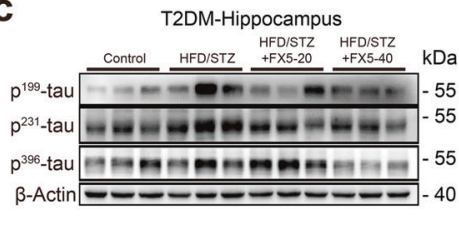

\section{g}

T2DM-Hippocampus

HFD/STZ+

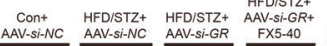

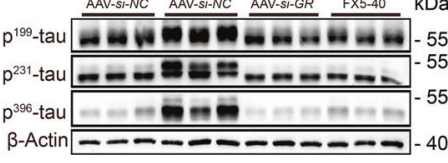

k

K T2DM-Hippocampus
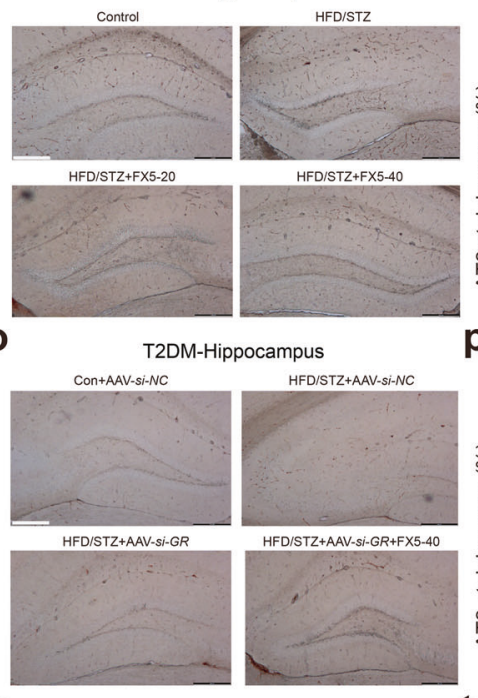

$\mathbf{S}$

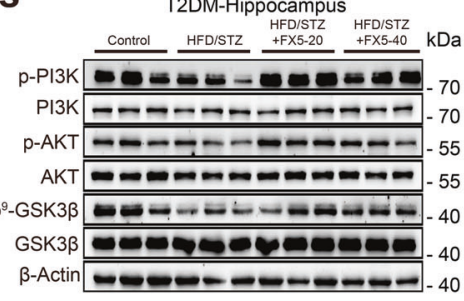

W

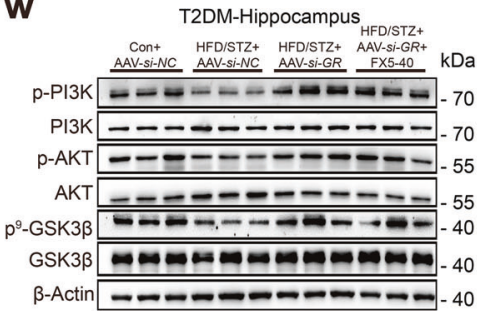

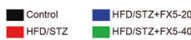

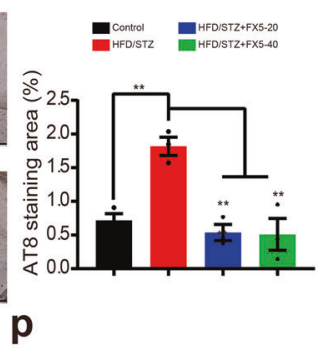

$p$
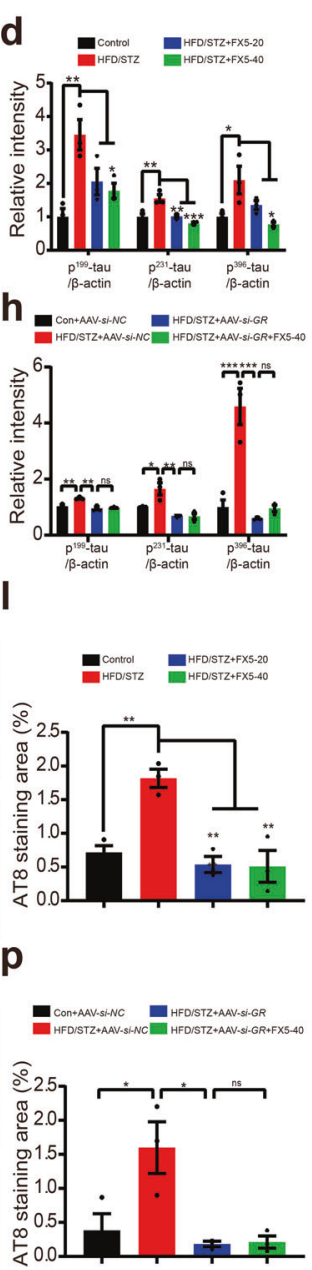

$\mathbf{t}$

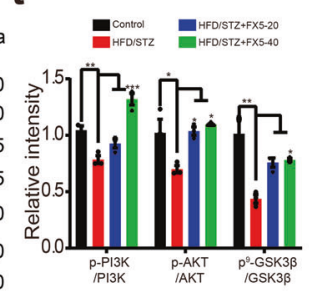

$\mathbf{X}$

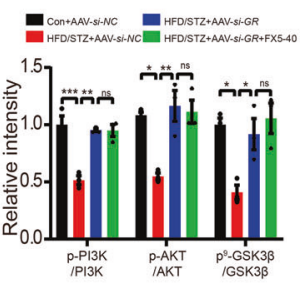

Fig. 6 FX5 attenuated tauopathy through GR/PI3K/AKT/GSK3 $\beta$ pathway in the hippocampus of diabetic mice. FX5 (20, $40 \mathrm{mg} / \mathrm{kg})$ or AAV-si-GR treatment repressed tau hyperphosphorylation in the hippocampus of (a, b, e, f) STZ and (c, d, g, h) HFD/STZ mice, and FX5 $(40 \mathrm{mg} / \mathrm{kg}$ ) had no impacts on tau hyperphosphorylation in (e, f) STZ + AAV-si-GR and (g, h) HFD/STZ + AAV-si-GR mice ( $n=3$ per group). FX5 $(20,40 \mathrm{mg} / \mathrm{kg})$ or AAV-si-GR treatment suppressed AT8 expression in the hippocampus of (i, j, $\mathbf{m}, \mathbf{n}) \mathrm{STZ}$ and $(\mathbf{k}, \mathbf{I}, \mathbf{o}, \mathbf{p})$ HFD/STZ mice, and FX5 $(40 \mathbf{~ m g} / \mathrm{kg})$ had no impacts on AT8 expression in $(\mathbf{m}, \mathbf{n})$ STZ + AAV-si-GR and (o, p) HFD/STZ + AAV-si-GR mice (positive AT8 protein was labeled brown, $n=3$ per group). Scale bar: $200 \mu \mathrm{m}$. FX5 $(20,40 \mathrm{mg} / \mathrm{kg})$ or AAV-si-GR treatment upregulated the levels of p-PI3K, p-AKT (Ser 473$)$ and p-GSK3 3 (Ser 9) in the hippocampus of $(\mathbf{q}, \mathbf{r}, \mathbf{u}, \mathbf{v})$ STZ and $(\mathbf{s}, \mathbf{t}, \mathbf{w}, \mathbf{x})$ HFD/STZ mice, and FX5 (40 mg/kg) had no impacts on these proteins in $(\mathbf{u}, \mathbf{v})$ STZ + AAV-si-GR and (w, x) HFD/STZ + AAV-si-GR mice $(n=3$ per group). $\beta$-Actin was used as loading control. $n=3$ independent experiments. Mean \pm SEM. One-way ANOVA followed by Dunnett's multiple comparison test. ${ }^{*} P<0.05,{ }^{* *} P<0.01,{ }^{* * *} P<0.001$ vs model or AAVsi-NC treated model group. 
a b -

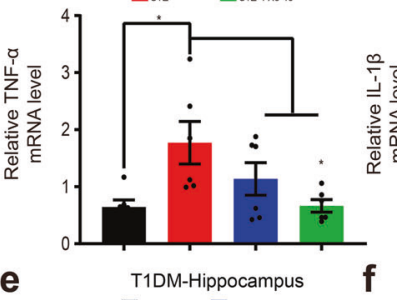

e

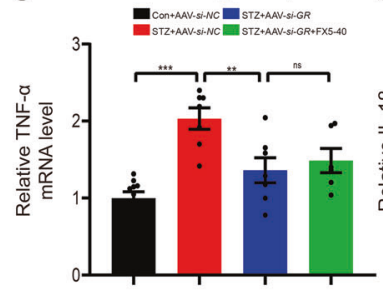

i

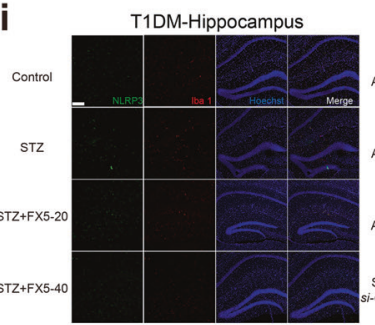

$\mathbf{k}$

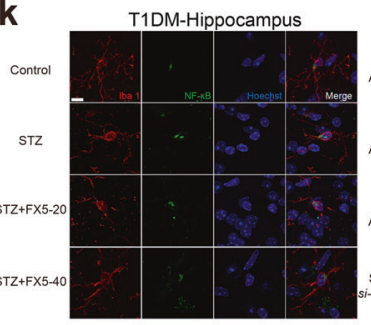

m

T1DM-Hippocampus

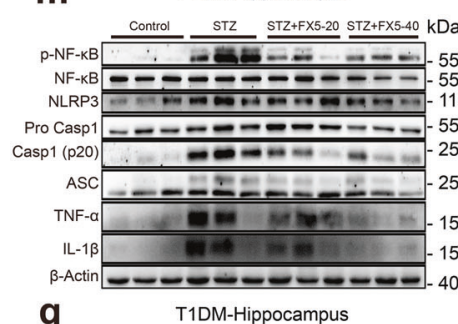

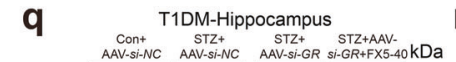

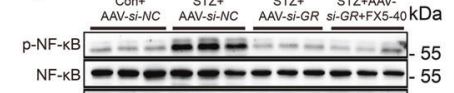

NLRP3

Pro Casp1 1

Casp1 (p20)

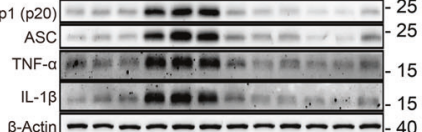

T1DM-Hippocampus

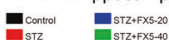

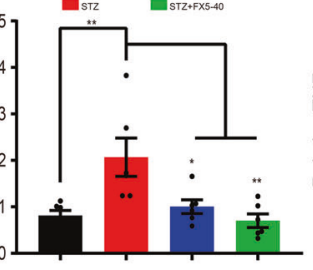

T1DM-Hippocampus

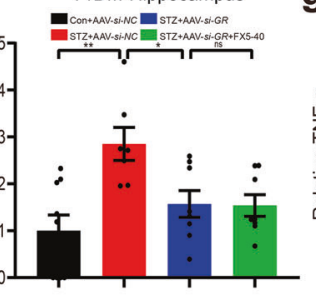

T1DM-Hippocampus

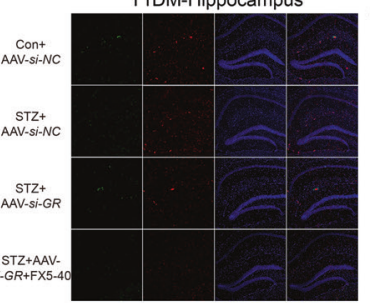

T1DM-Hippocampus

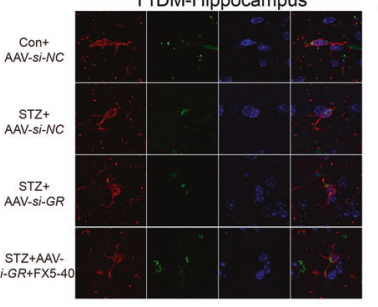

n

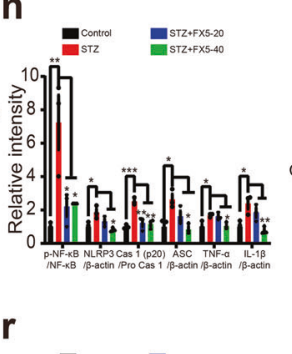

C

T2DM-Hippocampus

d

T2DM-Hippocampus

E

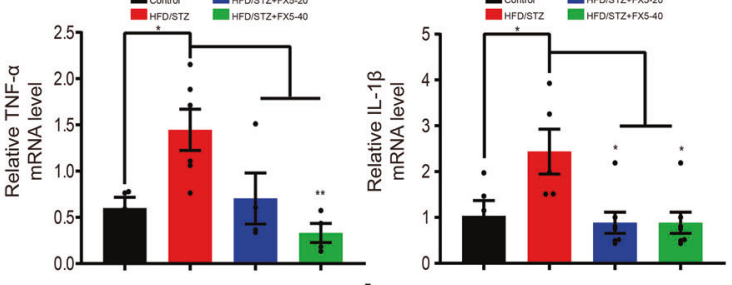

g

T2DM-Hippocampus h

T2DM-Hippocampus


T2DM-Hippocampus

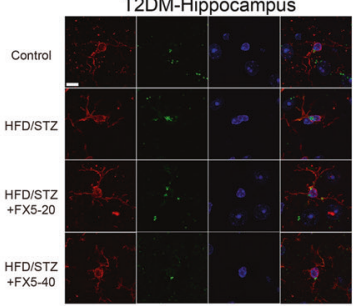

0

T2DM-Hippocampus
Pro Casp1

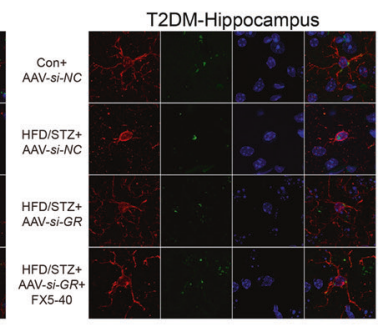

p

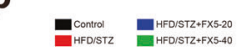

블

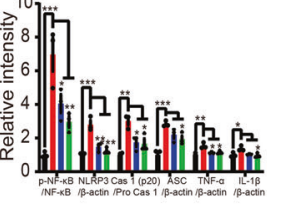

$t$

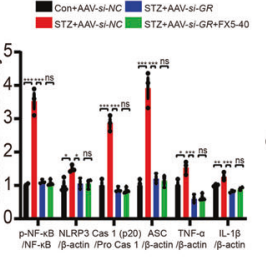

$\mathbf{S}$

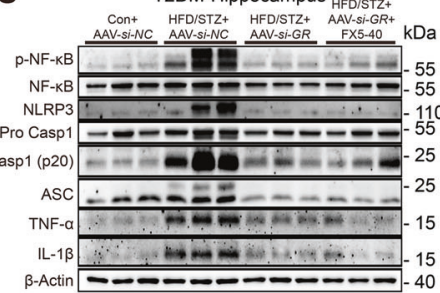

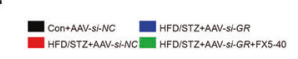

-

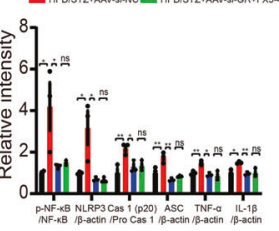

Fig. 7 FX5 repressed inflammation through GR/NF-KB/NLRP3/ASC/Caspase-1 pathway in the hippocampus of diabetic mice. FX5 (20, 40 $\mathrm{mg} / \mathrm{kg}$ ) or AAV-si-GR treatment attenuated the levels of TNF- $\alpha$ and IL-1 $\beta$ in the hippocampus of $(\mathbf{a}, \mathbf{b}, \mathbf{e}, \mathbf{f})$ STZ and (c, d, g, h) HFD/STZ mice, and FX5 $(40 \mathrm{mg} / \mathrm{kg}$ ) rendered no such effects in (e, f) STZ + AAV-si-GR and (g, h) HFD/STZ + AAV-si-GR mice ( $n \geq 4$ per group). FX5 (20, 40 mg/ $\mathrm{kg}$ ) or AAV-si-GR treatment repressed NLRP3 inflammasome, and FX5 (40 mg/kg) rendered no such an effect in (i) STZ + AAV-si-GR and (j) HFD/ STZ + AAV-si-GR mice ( $n \geq 8$ per group). Scale bar: $10 \mu \mathrm{m}$. FX5 $(20,40 \mathrm{mg} / \mathrm{kg})$ or AAV-si-GR treatment suppressed the nuclear translocation of NF-kB in microglia of diabatic mice, and FX5 $(40 \mathrm{mg} / \mathrm{kg})$ rendered no such an effect in (k) STZ + AAV-si-GR and (I) HFD/STZ + AAV-si-GR mice ( $n \geq 7$ per group). Scale bar: $10 \mu \mathrm{m}$. FX5 $(20,40 \mathrm{mg} / \mathrm{kg})$ or AAV-si-GR treatment suppressed the protein levels of p-NF- $\mathrm{kB}$, NLRP3, ASC, Caspase-1 (p20), TNF- $\alpha$ and IL-1 $\beta$ in the hippocampus of $(\mathbf{m}, \mathbf{n}, \mathbf{q}, \mathbf{r})$ STZ and $(\mathbf{o}, \mathbf{p}, \mathbf{s}, \mathbf{t})$ HFD/STZ mice, and FX5 (40 mg/kg) had no such effects in (q, r) STZ $+\mathrm{AAV}-\mathrm{si}-\mathrm{GR}$ and $(\mathbf{s}, \mathbf{t}) \mathrm{HFD} / \mathrm{STZ}+\mathrm{AAV}-\mathrm{si}-\mathrm{GR}$ mice $(n=3$ per group). $\beta$-Actin was used as loading control. $n=3$ independent experiments. Mean \pm SEM. One-way ANOVA followed by Dunnett's multiple comparison test. ${ }^{*} P<0.05$, ${ }^{* *} P<0.01,{ }^{* * *} P<0.001$ vs model or AAV-si-NC treated model group. 
NF-KB nuclear translocation in diabetic mice, and AAV-si-GR deprived FX5 of its inhibitory activity in AAV-si-GR-treated diabetic mice.

In addition, immunoblot assay results demonstrated that FX5 or AAV-si-GR treatment suppressed the protein levels of $\mathrm{p}-\mathrm{NF}-\mathrm{kB}$, NLRP3, ASC and Caspase-1 (P20) in the hippocampus and cortex of the diabetic mice, and AAV-si-GR injection deprived FX5 of its suppressive capability in AAV-si-GR-treated diabetic mice (Fig. 7m-t and Supplementary Fig. S6i-p). These results demonstrated that FX5 repressed NF-KB/NLRP3/ASC/Caspase-1 pathway in diabetic mice by antagonizing $G R$.

Collectively, FX5 repressed inflammation through GR/NF-KB/ NLRP3/ASC/Caspase-1 pathway in diabetic mice.

\section{DISCUSSION}

$\mathrm{DCl}$ is a chronic complication of diabetes with complicated pathogenesis. It was reported that $G R$ antagonists can improve $D C l$ in mice by repressing hippocampal $A \beta$ generation, neuroinflammation and apoptotic processes [25, 29, 66], but the detailed mechanisms are much needed. Here, we determined that small molecule FX5 as a non-steroidal GR antagonist effectively ameliorated learning and memory impairment in diabetic mice and the underlying mechanisms have been intensively investigated. Our results have highlighted the potential of FX5 in the treatment of $\mathrm{DCl}$.

In the brain, synaptic plasticity and integrity are critically responsible for cognition. BDNF is a widespread neurotrophin facilitating neuroprotection and synaptic interaction by binding its receptor TrkB, and GR regulates BDNF expression through directly binding to BDNF regulatory sequences [67]. Thus, our finding that FX5 efficiently ameliorated synaptic impairment through GR/ $\mathrm{BDNF} / \mathrm{TrkB} / \mathrm{CREB}$ pathway has clearly revealed the underlying mechanism for the beneficial effect of GR antagonist on synaptic protection.

Tau as a major microtubule-associated protein of normal mature neurons aggravates neurotoxicity by excessive phosphorylation resulting in cognitive dysfunction in diabetic mice [68]. According to the published report, GR could decrease PI3K activity in skeletal muscle through a direct inhibitory interaction between PI3K p85 regulatory subunit and GR [69]. In diabetic pathology, GCs suppress the expression and activity of PI3K and AKT [70, 71], triggering the activation of GSK3 $\beta$ that is responsible for tau phosphorylation [72]. Subsequently, tau phosphorylation has been also identified to induce ER stress, as indicated by the study on tau transgenic mice that the interaction between tau and ER membrane impaired ER-associated degradation and activated unfolded protein response (UPR) [73, 74] inducing apoptosis [54]. Here, we determined that FX5 protected against neuronal apoptosis by repressing GR/PI3K/AKT/GSK3 $\beta$ pathway-mediated tauopathy and subsequent ER stress, strongly supporting that antagonizing GR might be a promising therapeutic strategy in treating neuronal apoptosis and its related degenerative diseases.

$\mathrm{DCl}$ is highly correlated with inflammation [75]. Although GR agonist exhibits anti-inflammation effect, chronic GCs exposure may increase NF-KB activation [76], inducing the expressions of TNF-a [77] and NLRP3 [78]. Here, FX5 as a non-steroidal GR antagonist was determined to efficiently repress inflammation through GR/NF-KB/NLRP3/ASC/Caspase-1 pathway. To our knowledge, our work might be the first report on the mechanism underlying the regulation of $\mathrm{GR}$ against inflammation in $\mathrm{DCl}$.

In summary, we determined that small molecule FX5 as a nonsteroidal GR antagonist efficiently ameliorated learning and memory impairment in diabetic mice. The underlying mechanisms have been intensively investigated. As summarized in Fig. 8, FX5 ameliorated synaptic impairment through GR/BDNF/TrkB/CREB pathway, protected against neuronal apoptosis by repressing GR/ $\mathrm{PI} 3 \mathrm{~K} / \mathrm{AKT} / \mathrm{GSK} 3 \beta$ pathway-mediated tauopathy and subsequent ER stress and repressed inflammation through GR/NF-KB/NLRP3/ASC/

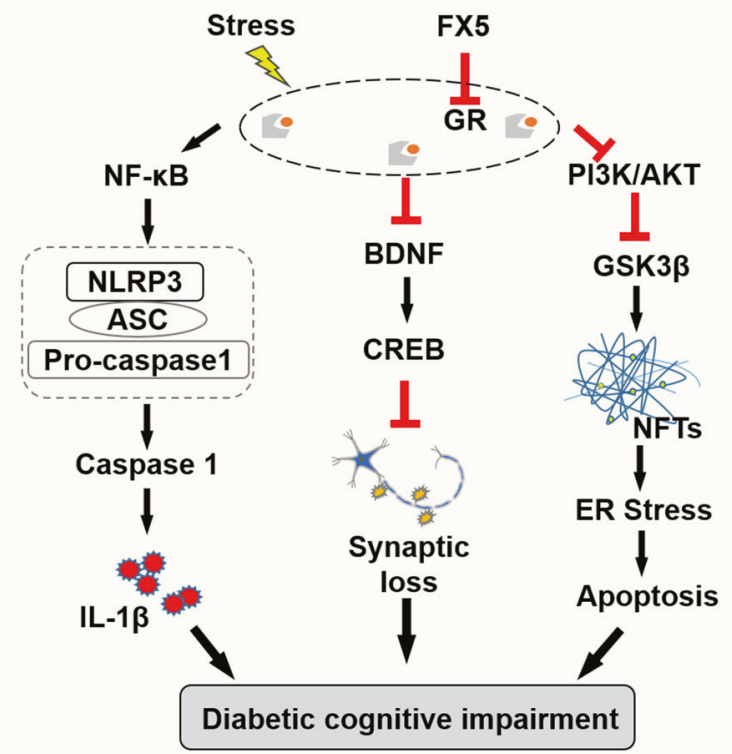

Fig. 8 The mechanisms underlying the protective effects of FX5 against diabetic cognitive impairment. FX5 as a non-steroidal GR antagonist ameliorated synaptic impairment through GR/BDNF/TrkB/ CREB pathway, protected against neuronal apoptosis by repressing GR/PI3K/AKT/GSK3 $\beta$ pathway-mediated tauopathy and subsequent ER stress, and repressed inflammation through GR/NF-KB/NLRP3/ASC/ Caspase-1 pathway.

Caspase-1 pathway. Our work has strongly addressed the potency of non-steroidal GR antagonist in the amelioration of $\mathrm{DCl}$ and highlighted the potential of FX5 in the treatment of this disease, although further research (e.g., structure optimization, toxicity, and oral availability evaluation) is much needed before its clinical application.

\section{ACKNOWLEDGEMENTS}

This work was supported by Innovative Research Team of Six Talent Peaks Project in Jiangsu Province (grant number TD-SWYY-013), the National Natural Science Foundation for Young Scientists of China (grant number 81703806), the Natural Science Foundation for Young Scientists of Nanjing University of Chinese Medicine (grant number NZY81703806), the Open Project of Chinese Materia Medica First-Class Discipline of Nanjing University of Chinese Medicine (grant number 2020YLXK018), and Postgraduate Research \& Practice Innovation Program of Jiangsu Province (grant number KYCX21 1737).

\section{AUTHOR CONTRIBUTIONS}

DYZ and XS designed the study. XS reviewed the paper. YNZ synthetized FX5. DYZ, JL, RX, JZY, XRM, XNOY, QYY, RFN, TZ and YDC performed the animal and cell experiments. DYZ and YL analyzed and interpreted data. DYZ wrote the paper. XS and WJL are the guarantors of this work and have full access to all data in the study and take responsibility for the integrity of the data and the accuracy of the data analysis. All authors approved the paper.

\section{ADDITIONAL INFORMATION}

Supplementary information The online version contains supplementary material available at https://doi.org/10.1038/s41401-022-00884-9.

Competing interests: The authors declare no competing interests.

\section{REFERENCES}

1. Biessels GJ, Whitmer RA. Cognitive dysfunction in diabetes: how to implement emerging guidelines. Diabetologia. 2020;63:3-9. 
2. Ayelign $B$, Negash $M$, Andualem $H$, Wondemagegn $T$, Kassa $E$, Shibabaw $T$, et al. Association of IL-10 (- $1082 \mathrm{~A} / \mathrm{G})$ and IL-6 $(-174 \mathrm{G} / \mathrm{C})$ gene polymorphism with type 2 diabetes mellitus in Ethiopia population. BMC Endocr Disord. 2021;21:70-8.

3. Moore EM, Mander AG, Ames D, Kotowicz MA, Carne RP, Brodaty $H$, et al. Increased risk of cognitive impairment in patients with diabetes is associated with metformin. Diabetes Care. 2013:36:2981-7.

4. Rahmati M, Keshvari M, Mirnasouri R, Chehelcheraghi F. Exercise and Urtica dioica extract ameliorate hippocampal insulin signaling, oxidative stress, neuroinflammation, and cognitive function in STZ-induced diabetic rats. Biomed Pharmacother. 2021;139:111577-86.

5. Suzumura A, Kaneko H, Funahashi Y, Takayama K, Nagaya M, Ito S, et al. n-3 fatty acid and its metabolite 18-HEPE ameliorate retinal neuronal cell dysfunction by enhancing Müller BDNF in diabetic retinopathy. Diabetes. 2020;69:724-35.

6. Spangenberg EE, Green KN. Inflammation in Alzheimer's disease: Lessons learned from microglia-depletion models. Brain Behav Immun. 2017;61:1-11.

7. Akhtar A, Sah SP. Insulin signaling pathway and related molecules: role in neurodegeneration and Alzheimer's disease. Neurochem Int. 2020;135:104707-18.

8. Kitagishi Y, Nakanishi A, Ogura Y, Matsuda S. Dietary regulation of PI3K/AKT/GSK3beta pathway in Alzheimer's disease. Alzheimers Res Ther. 2014;6:35-42.

9. Hussain G, Wang J, Rasul A, Anwar H, Imran A, Qasim M, et al. Role of cholesterol and sphingolipids in brain development and neurological diseases. Lipids Health Dis. 2019;18:26-38

10. Nemeth $\mathrm{CL}$, Neigh $\mathrm{GN}$. Microemboli alter the acute stress response and cause prolonged expression of MCP-1 in the hippocampus. Psychoneuroendocrinology. 2015;54:71-7.

11. Hardy R, Rabbitt EH, Filer A, Emery P, Hewison M, Stewart PM, et al. Local and systemic glucocorticoid metabolism in inflammatory arthritis. Ann Rheum Dis. 2008;67:1204-10.

12. Ahmed A, Schmidt C, Brunner T. Extra-adrenal glucocorticoid synthesis in the intestinal mucosa: between immune homeostasis and immune escape. Front Immunol. 2019;10:1438-57.

13. Arnett MG, Muglia LM, Laryea G, Muglia L. Genetic approaches to hypothalamicpituitary-adrenal axis regulation. Neuropsychopharmacology. 2016;41:245-60.

14. Messier C. Impact of impaired glucose tolerance and type 2 diabetes on cognitive aging. Neurobiol Aging. 2005;26:26-30.

15. Desrocher M, Rovet J. Neurocognitive correlates of type 1 diabetes mellitus in childhood. Child Neuropsychol. 2004;10:36-52.

16. Chen YT, Hu Y, Yang QY, Son JS, Liu XD, de Avila JM, et al. Excessive glucocorticoids during pregnancy impair fetal brown fat development and predispose offspring to metabolic dysfunctions. Diabetes. 2020;69:1662-74.

17. Strachan MW, Reynolds RM, Frier BM, Mitchell RJ, Price JF. The role of metabolic derangements and glucocorticoid excess in the aetiology of cognitive impairment in type 2 diabetes. Implications for future therapeutic strategies. Diabetes Obes Metab. 2009;11:407-14.

18. Choi GE, Han HJ. Glucocorticoid impairs mitochondrial quality control in neurons Neurobiol Dis. 2021;152:105301-12.

19. Vyas S, Rodrigues AJ, Silva JM, Tronche F, Almeida OFX, Sousa N, et al. Chronic stress and glucocorticoids: from neuronal plasticity to neurodegeneration. Neural Plast. 2016;2016:6391686-6391701.

20. Monteiro-Fernandes D, Sousa N, Almeida OFX, Sotiropoulos I. Sex hormone depletion augments glucocorticoid induction of Tau hyperphosphorylation in male rat brain. Neuroscience. 2021;454:140-50.

21. Wosiski-Kuhn M, Erion JR, Gomez-Sanchez EP, Gomez-Sanchez CE, Stranahan AM. Glucocorticoid receptor activation impairs hippocampal plasticity by suppressing BDNF expression in obese mice. Psychoneuroendocrinology. 2014;42:165-77.

22. Zhang B, Zhang Y, Wu W, Xu T, Yin Y, Zhang J, et al. Chronic glucocorticoid exposure activates BK-NLRP1 signal involving in hippocampal neuron damage. $J$ Neuroinflammation. 2017;14:139-52.

23. Dalle H, Garcia M, Antoine B, Boehm V, Do $\Pi \mathrm{H}$, Buyse $M$, et al. Adipocyte glucocorticoid receptor deficiency promotes adipose tissue expandability and improves the metabolic profile under corticosterone exposure. Diabetes. 2019:68:305-17.

24. Riedemann T, Patchev AV, Cho K, Almeida OFX. Corticosteroids: way upstream. Mol Brain. 2010;3:2-22.

25. Zuo ZF, Wang W, Niu L, Kou ZZ, Zhu C, Wang W, et al. RU486 (mifepristone) ameliorates cognitive dysfunction and reverses the down-regulation of astrocytic $\mathrm{N}$-myc downstream-regulated gene 2 in streptozotocin-induced type- 1 diabetic rats. Neuroscience. 2011;190:156-65.

26. Castinetti F, Fassnacht $M$, Johanssen $S$, Terzolo $M$, Bouchard $P$, Chanson $P$, et al. Merits and pitfalls of mifepristone in Cushing's syndrome. Eur J Endocrinol. 2009;160:1003-10.

27. Yuen KCJ, Moraitis A, Nguyen D. Evaluation of evidence of adrenal insufficiency in trials of normocortisolemic patients treated with mifepristone. J Endocr Soc. 2017;1:237-46.
28. Macnaughton $\mathrm{H}$, Nothnagle $\mathrm{M}$, Early J. Mifepristone and misoprostol for early pregnancy loss and medication abortion. Am Fam Phys. 2021;103:473-80.

29. Pineau F, Canet G, Desrumaux C, Hunt H, Chevallier N, Ollivier M, et al. New selective glucocorticoid receptor modulators reverse amyloid-beta peptideinduced hippocampus toxicity. Neurobiol Aging. 2016;45:109-22.

30. Xu X, Chen Y, Zhu D, Zhao T, Xu R, Wang J, et al. FX5 as a non-steroidal GR antagonist improved glucose homeostasis in type 2 diabetic mice via GR/ HNF4alpha/miR-122-5p pathway. Aging (Albany NY). 2020;13:2436-58.

31. Lu J, Zhang C, Lv J, Zhu X, Jiang X, Lu W, et al. Antiallergic drug desloratadine as a selective antagonist of $5 \mathrm{HT} 2 \mathrm{~A}$ receptor ameliorates pathology of Alzheimer's disease model mice by improving microglial dysfunction. Aging Cell. 2021;20: e13286.

32. Liu $Q$, Zhang $Y$, Lin $Z$, Shen $H$, Chen $L$, $H u$, et al. Danshen extract 15,16dihydrotanshinone I functions as a potential modulator against metabolic syndrome through multi-target pathways. J Steroid Biochem Mol Biol 2010;120:155-63.

33. Yao XG, Xu X, Wang GH, Lei $M$, Quan LL, Cheng $Y H$, et al. BBT improves glucose homeostasis by ameliorating beta-cell dysfunction in type 2 diabetic mice. J Endocrinol. 2015;224:327-41.

34. Zhu X, Chen Y, Xu X, Xu X, Lu Y, Huang X, et al. SP6616 as a Kv2.1 inhibitor efficiently ameliorates peripheral neuropathy in diabetic mice. EBioMedicine. 2020;61:103061-77.

35. Kim DY, Kim SR, Jung UJ. Myricitrin ameliorates hyperglycemia, glucose intolerance, hepatic steatosis, and inflammation in high-fat diet/streptozotocin-induced diabetic mice. Int J Mol Sci. 2020;21:1870-83.

36. Mathiesen SN, Lock JL, Schoderboeck L, Abraham WC, Hughes SM. CNS transduction benefits of AAV-PHP.eB over AAV9 are dependent on administration route and mouse strain. Mol Ther Methods Clin Dev. 2020;19:447-58.

37. Mehta BK, Banerjee S. Characterization of cognitive impairment in type 2 diabetic rats. Indian J Pharmacol Sci. 2017;79:785-93.

38. Boyer F, Jaouen F, Ibrahim EC, Gascon E. Deficits in social behavior precede cognitive decline in middle-aged mice. Front Behav Neurosci. 2019;13:55-66.

39. Christakis DA, Ramirez JSB, Ferguson SM, Ravinder S, Ramirez JM. How early media exposure may affect cognitive function: a review of results from observations in humans and experiments in mice. Proc Natl Acad Sci USA 2018;115:9851-8.

40. Wahl D, Coogan SC, Solon-Biet SM, de Cabo R, Haran JB, Raubenheimer D, et al. Cognitive and behavioral evaluation of nutritional interventions in rodent models of brain aging and dementia. Clin Inter Aging. 2017;12:1419-28.

41. Guo XD, Sun GL, Zhou TT, Wang YY, Xu X, Shi XF, et al. LX2343 alleviates cognitive impairments in $A D$ model rats by inhibiting oxidative stress-induced neuronal apoptosis and tauopathy. Acta Pharmacol Sin. 2017;38:1104-19.

42. Tang $X$, Shen $H$, Chen J, Wang $X$, Zhang $Y$, Chen LL, et al. Activating transcription factor 6 protects insulin receptor from ER stress-stimulated desensitization via p42/44 ERK pathway. Acta Pharmacol Sin. 2011;32:1138-47.

43. Xu X, Xu X, Liu P, Zhu ZY, Chen J, Fu HA, et al. Structural basis for small molecule NDB (N-Benzyl-N-(3-(tert-butyl)-4-hydroxyphenyl)-2,6-dichloro-4-(dimethylamino) Benzamide) as a selective antagonist of farnesoid $X$ receptor alpha (FXRalpha) in stabilizing the homodimerization of the receptor. J Biol Chem. 2015;290:19888-99.

44. Li YJ, Li XR, Yang J, He YK. Natural antisense transcripts of MIR398 genes suppress microR398 processing and attenuate plant thermotolerance. Nat Commun. 2020;11:5351-64.

45. Reno CM, Puente EC, Sheng Z, Daphna-lken D, Bree AJ, Routh VH, et al. Brain GLUT4 knockout mice have impaired glucose tolerance, decreased insulin sensitivity, and impaired hypoglycemic counterregulation. Diabetes. 2017;66:587-97.

46. Patil S, Melrose J, Chan C. Involvement of astroglial ceramide in palmitic acidinduced Alzheimer-like changes in primary neurons. Eur J Neurosci. 2007;26:2131-41.

47. Park HR, Kim JY, Park KY, Lee J. Lipotoxicity of palmitic Acid on neural progenitor cells and hippocampal neurogenesis. Toxicol Res. 2011;27:103-10.

48. Fatima S, Hu XJ, Gong RH, Huang $\mathrm{CH}$, Chen MT, Wong HLX, et al. Palmitic acid is an intracellular signaling molecule involved in disease development. Cell Mol Life Sci. 2019;76:2547-57.

49. Nemeth M, Schuster D, Millesi E, Wallner B. Dietary fatty acids modulate cortisol concentrations and social dominance during social confrontations in adolescent male guinea pigs. Psychoneuroendocrinology. 2021;123:105045-55.

50. Batista AF, Forny-Germano L, Clarke JR, Silva NMLE, Brito-Moreira J, Boehnke SE, et al. The diabetes drug liraglutide reverses cognitive impairment in mice and attenuates insulin receptor and synaptic pathology in a non-human primate model of Alzheimer's disease. J Pathol. 2018;245:85-100.

51. Stragier E, Martin V, Davenas E, Poilbout C, Mongeau R, Corradetti R, et al. Brain plasticity and cognitive functions after ethanol consumption in C57BL/6J mice. Transl Psychiat. 2015;5:e696. 
52. Yao L, Li M, Sun S, Xu M, Yu S, Zhang Z, et al. Multimodal brain imaging effect of "Adjust Zang-fu and Arouse Spirit" electroacupuncture on diabetic cognitive impairment: study protocol of a randomized, sham-controlled pilot trial. Trials. 2021;22:847-58

53. Zhang XM, Xu LH, He DQ, Ling SC. Endoplasmic reticulum stress-mediated hippocampal neuron apoptosis involved in diabetic cognitive impairment. Biomed Res Int. 2013;2013:924327-36.

54. Deegan S, Saveljeva S, Gorman AM, Samali A. Stress-induced self-cannibalism: on the regulation of autophagy by endoplasmic reticulum stress. Cell Mol Life Sci. 2013;70:2425-41.

55. Jinawong K, Apaijai N, Wongsuchai S, Pratchayasakul W, Chattipakorn N, Chattipakorn SC. Necrostatin-1 mitigates cognitive dysfunction in prediabetic rats with no alteration in insulin sensitivity. Diabetes. 2020;69:1411-23.

56. Iqbal K, Liu F, Gong CX, Alonso Adel C, Grundke-lqbal I. Mechanisms of tauinduced neurodegeneration. Acta Neuropathol. 2009;118:53-69.

57. Kim I, Xu W, Reed JC. Cell death and endoplasmic reticulum stress: disease relevance and therapeutic opportunities. Nat Rev Drug Discov. 2008;7:1013-30.

58. Heyer EJ, Mergeche JL, Bruce SS, Connolly ES. Inflammation and cognitive dysfunction in type 2 diabetic carotid endarterectomy patients. Diabetes Care. 2013;36:3283-6.

59. Afonina IS, Zhong ZY, Karin M, Beyaert R. Limiting inflammation-the negative regulation of NF-kappa B and the NLRP3 inflammasome. Nat Immunol. 2017;18:861-9.

60. Groslambert M, Py BF. Spotlight on the NLRP3 inflammasome pathway. J Inflamm Res. 2018;11:359-74.

61. Ambrosini G, Do C, Tycko B, Realubit RB, Karan C, Musi E, et al. Inhibition of NFkappaB-dependent signaling enhances sensitivity and overcomes resistance to BET inhibition in uveal melanoma. Cancer Res. 2019;79:2415-25.

62. Paltian JJ, dos Reis AS, de Oliveira RL, da Fonseca CAR, Domingues WB, Dellagostin EN, et al. The anxiolytic effect of a promising quinoline containing selenium with the contribution of the serotonergic and GABAergic pathways: modulation of parameters associated with anxiety in mice. Behav Brain Res. 2020;393:112797-807.

63. Wang YD, Taylor E, Zikopoulos B, Seta F, Huang NS, Hamilton JA, et al. Aginginduced microbleeds of the mouse thalamus compared to sensorimotor and memory defects. Neurobiol Aging. 2021;100:39-47.

64. Ouchi Y, Banno Y, Shimizu Y, Ando S, Hasegawa H, Adachi K, et al. Reduced adult hippocampal neurogenesis and working memory deficits in the Dgcr8-deficient mouse model of 22q11.2 deletion-associated schizophrenia can be rescued by IGF2. J Neurosci. 2013;33:9408-19.

65. Ge YA, Dong ZF, Bagot RC, Howland JG, Phillips AG, Wong TP, et al. Hippocampal long-term depression is required for the consolidation of spatial memory. Proc Natl Acad Sci USA. 2010;107:16697-702.

66. Dey A, Hao S, Wosiski-Kuhn M, Stranahan AM. Glucocorticoid-mediated activation of GSK3beta promotes tau phosphorylation and impairs memory in type 2 diabetes. Neurobiol Aging. 2017;57:75-83.

67. Chen $\mathrm{H}$, Lombes $\mathrm{M}$, Le Menuet D. Glucocorticoid receptor represses brain-derived neurotrophic factor expression in neuron-like cells. Mol Brain. 2017;10:12-28.
68. d'Orange M, Auregan G, Cheramy D, Gaudin-Guerif M, Lieger S, Guillermier M, et al. Potentiating tangle formation reduces acute toxicity of soluble tau species in the rat. Brain. 2018;141:535-49.

69. Hu ZY, Wang HL, Lee IH, Du J, Mitch WE. Endogenous glucocorticoids and impaired insulin signaling are both required to stimulate muscle wasting under pathophysiological conditions in mice. J Clin Invest. 2009;119:3059-69.

70. Liu J, Han YS, Liu L, Tang L, Yang H, Meng P, et al. Abnormal Glu/mGluR2/3/PI3K pathway in the hippocampal neurovascular unit leads to diabetes-related depression. Neural Regen Res. 2021;16:727-33.

71. Zheng B, Ohkawa S, Li H, Roberts-Wilson TK, Price SR. FOXO3a mediates signaling crosstalk that coordinates ubiquitin and atrogin-1/MAFbx expression during glucocorticoid-induced skeletal muscle atrophy. FASEB J. 2010;24:2660-9.

72. Sen T, Saha P, Jiang T, Sen N. Sulfhydration of AKT triggers Tau-phosphorylation by activating glycogen synthase kinase 3 beta in Alzheimer's disease. Proc Natl Acad Sci USA. 2020;117:4418-27.

73. Abisambra JF, Jinwal UK, Blair L, O'Leary JC 3rd, Li Q, Brady S, et al. Tau accumulation activates the unfolded protein response by impairing endoplasmic reticulum-associated degradation. J Neurosci. 2013;33:9498-507.

74. Hashimoto S, Saido TC. Critical review: involvement of endoplasmic reticulum stress in the aetiology of Alzheimer's disease. Open Biol. 2018;8:180024-39.

75. Hosny SS, Bahaaeldin AM, Khater MS, Bekhet MM, Hebah HA, Hasanin GA. Role of inflammatory markers in elderly type 2 diabetic patients with mild cognitive impairment. Curr Diabetes Rev. 2019;15:247-53.

76. Munhoz CD, Lepsch LB, Kawamoto EM, Malta MB, Lima Lde S, Avellar MC, et al. Chronic unpredictable stress exacerbates lipopolysaccharide-induced activation of nuclear factor-kappaB in the frontal cortex and hippocampus via glucocorticoid secretion. J Neurosci. 2006;26:3813-20.

77. Attiq A, Yao LJ, Afzal S, Khan MA. The triumvirate of NF-kappaB, inflammation and cytokine storm in COVID-19. Int Immunopharmacol. 2021;101:108255-68.

78. Liu T, Zhang L, Joo D, Sun SC. NF-kappaB signaling in inflammation. Signal Transduct Target Ther. 2017;2:e17023.

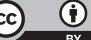

Open Access This article is licensed under a Creative Commons Attribution 4.0 International License, which permits use, sharing, adaptation, distribution and reproduction in any medium or format, as long as you give appropriate credit to the original author(s) and the source, provide a link to the Creative Commons license, and indicate if changes were made. The images or other third party material in this article are included in the article's Creative Commons license, unless indicated otherwise in a credit line to the material. If material is not included in the article's Creative Commons license and your intended use is not permitted by statutory regulation or exceeds the permitted use, you will need to obtain permission directly from the copyright holder. To view a copy of this license, visit http://creativecommons. org/licenses/by/4.0/.

(c) The Author(s) 2022 